\title{
Knitting Ourselves Into Being: the case of labour and hip domesticity on the social network Ravelry.com
}

\begin{abstract}
By
Maria Hellstrom

A thesis submitted to the Victoria University of Wellington in fulfilment of the requirements for the degree of Master of Arts in Media Studies
\end{abstract}

Victoria University of Wellington

2013 


\section{Table of Contents}

Chapter 1 Introducing Ravelry

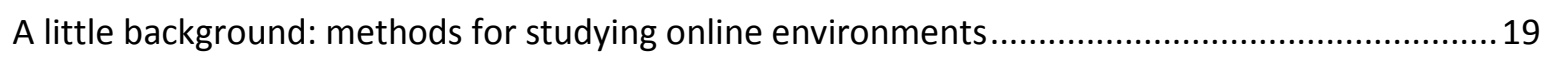

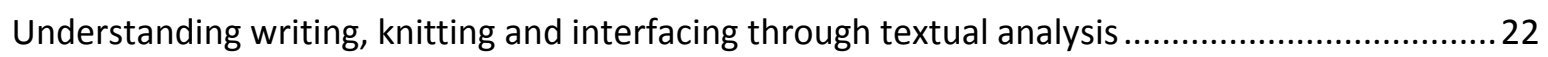

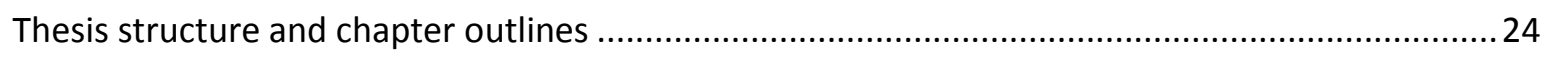

Chapter 2 - Finding the work in social network: an overview of social media labour...........................28

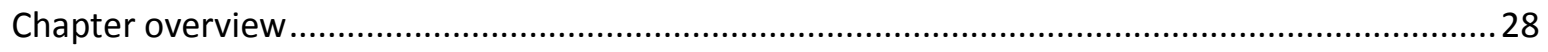

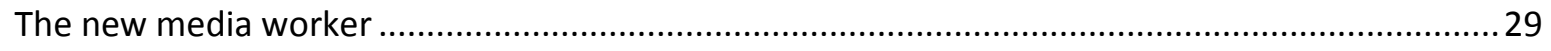

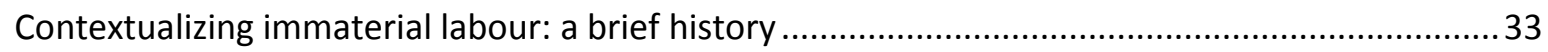

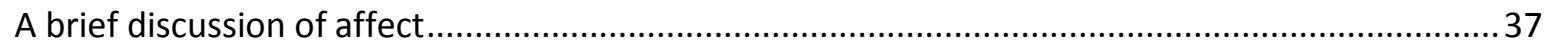

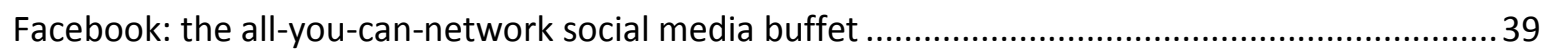

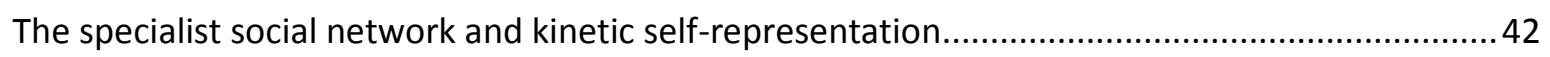

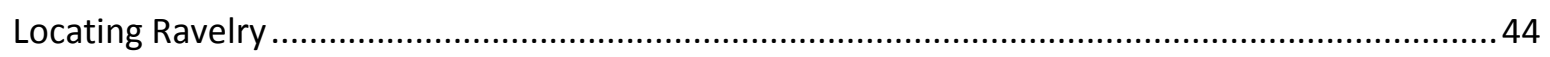

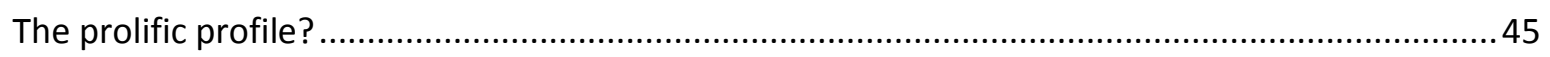

Chapter 3 - Hip domesticity and the cloak of affect ….....................................................................5

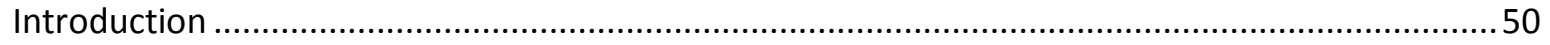

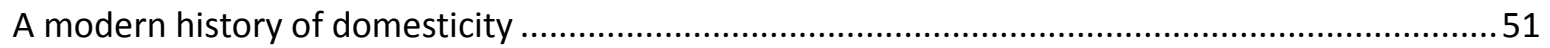

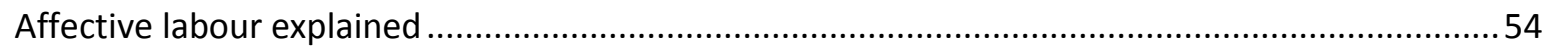

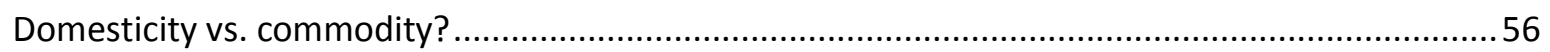

Hip home ec: from cyberfeminist resistance to hip domesticity ....................................................58 
Gender: the elephant in Ravelry's living room 61

I am what I am, not what I knit. Replicating one's offline social reality through online taste performance... 63

Hang on, travelling woman: grounding taste performance and affect in a finished object... .65

A critique of affect and immateriality.

Conclusion 69

Chapter 4 - Crafty commodities: buying, selling and knitting oneself into being.... .73

Introduction .73

Recapping Ravelry's spaces of interaction. .76

What's in your stash? Ravelry's user-driven yarn marketplace and discourses of exclusivity........ .79

The micro-economies of Ravelry: enmeshing handicrafts and capital. .84

The cloak of affect: Yarnies and enforced entrepreneurship .90

Incorporating materiality: Ravelry's contribution to theory .92

Conclusion .94

Overall conclusions .96 


\section{Abstract \\ Knitting Ourselves Into Being: the case of labour and hip domesticity on the social network Ravelry.com}

Thinking of social media participation in terms of doing work may seem a strange proposition. Yet, social network and handicrafts website Ravelry.com requires a great deal of labour from its members. From painstakingly hand-knitting fuzzy objects to photographing, recording and sharing these objects online, Ravelers must supply evidence of their hard work in order to fully participate in this online community. On Ravelry, "writing oneself into being" (Sundén 2002), or performing one's self through a textual medium, encompasses much more than simply writing. One must knit oneself into being too. Social capital is then accumulated through extensive cataloguing of handmade items. These 'finished objects' of knitting and crochet are imbued with affective meaning as tokens of nurturing and gift-giving, consistent with a historicity in which handicrafts like knitting have been associated with gendered care-work. Yet, much of Ravelry's activity centres on commodity exchange. Displaying commodity ownership is as important as displaying evidence of labour for the accrual of social capital. Recording and displaying domesticity as both acts of labour and acts of consumption fit within a wider trend of hip domesticity, where demonstrating one's domesticity has become a facet of popular culture. This project examines Ravelry.com's emphasis on the placement of a physical object between the self and the social network. The thesis argues that this incorporation of material objects into the structure of a social network challenges notions of disembodiment and immateriality. Ravelry.com demonstrates the need for a discussion of social media participant labour which goes beyond the immaterial and affective. 


\section{Chapter 1 - Introducing Ravelry}

\section{Welcome to the social network, where your knitting matters}

Traditional domestic arts such as knitting and crochet are perhaps not the associations which first spring to mind when talking about social networks and new media technologies. Yet, communities surrounding these traditional handicrafts flourish online. The fibrecrafts-oriented website Ravelry.com ("a site for knitters and crocheters") is prominent within these communities, and within this site, and others like it, a particular social network dynamic has emerged. At a cursory glance, the Ravelry world may seem straight-forward: it is a one-stop shop for the resources required for knitting or crocheting, and additionally fulfils the expectations of social network functions so prevalent within contemporary online mediascapes. Yet, an interesting moment occurs when attempting to access Ravelry's social network. It appears that simply identifying oneself as a knitter or crocheter (as one evidently is when signing up to such a niche site) is not in itself sufficient for gaining the social capital necessary for full entry into Ravelry as community. It is not adequate to state that one is a knitter or crocheter - one must prove it through acts of labour and documentation.

On Ravelry.com, members not only interact with each other through disembodied, immaterial space, they must also produce 'finished objects' (completed items of knitting or crochet) and present evidence of these objects in order to fully access Ravelry's social space. This evidence is important, as without it, Ravelers may struggle to receive the responses they hope for when posting to the site. For example, in the Ravelry forums members are expected to provide proof of their labour, particularly in the form of pictures of objects they have knitted, in order to be fully listened to by other forum members. A Raveler who posts in the forums asking for help with a knitting project, but who does not provide pictorial evidence of this project or who does not already have an established posting history, will often be asked for pictures of the object before adequate help is given. ${ }^{1}$ This is partly a practical concern as a picture of the problem can make it easier to troubleshoot, but it is also a matter of adhering to community norms. Similarly, posts simply stating that a project has been completed without supplying evidence in the form of pictures receive fewer responses than posts 
displaying images of completed projects. This potential withholding of help if community norms are not obeyed seems to run counter to the site's overall 'warm fuzziness". The translation of these material arts into technocultural contexts has brought with it a dynamic in which participation in Ravelry's online, disembodied community requires an anchoring in the offline, manual production of knitted or crocheted goods in order to maximise social capital. This offline anchoring contrasts with assumptions in scholarly literature about online environments as disembodied space. Moreover, the finished object becomes an important part of a Ravelry member's self-representation, as finished objects are imbued with varying degrees of social meaning and capital depending on a complex set of guiding parameters based on popularity, interactivity, intertextuality and precedent. In other words, not only is the act of knitting or crochet important for self-representation and social standing within Ravelry, the produced objects should adhere to community norms and tastes in order to maximize social capital. Placing a physical thing between self and participation in a social network is intriguing as it calls into question assumptions about online activity as disembodied or immaterial.

Ravelry.com is a website which encompasses handicrafts resources (both free and commercial) and social network functions. These social network functions are dissimilar to many other social networks as they utilize an architecture and logic wherein a member is expected to place evidence of an actual physical thing, a 'finished object', between his- or herself and his or her participation in the social network. Importantly, this incorporates an offline phenomenon into the process of what Sundén has referred to as the process, common on social networks, of writing oneself into being (Sundén 88), and requires an extensive labour-investment on the part of the user. Elements of Ravelry's labour-focused social dynamic can be observed in any social network where members produce digitized mirrors of their offline lives, through text, images or whatever format the social network demands. Ravelry therefore highlights an explicit connection between labour and social media participation, a phenomenon worthy of more scholarly attention. 


\section{We need to talk about labour}

Social networks have in recent years received a great deal of attention within a range of academic disciplines. It could be argued that social media has become ubiquitous to the point where its mere presence is no longer novel, and it has become both necessary and possible to delve deeper into the diverse structures and effects of social networks. A solid foundation of scholarly work dealing in various ways with how, why and where ICTs intersect with our everyday lives has already been established, with work ranging from the very early days of studying social networks which focused on the broader strokes of social networking technologies themselves (see, for example, "Studying Online Social Networks" by Garton et al., 1997) to in-depth case studies of social dynamics within specific, closed networks like Jenny Sundén's ethnographic study of selected Multi-User Dungeon users in “Cyberbodies: Writing Gender in Digital SelfPresentations" (Digital Borderlands 2002). Journals like Convergence (est. 1995) and First Monday (est. 1995) supply a steady stream of innovative, thought-provoking and thorough investigations into the social worlds online. Use of social networks, particularly behemoths like Facebook, has become so commonplace that scholars have observed that there can be perceived danger in not participating in online networks through the loss of social capital, particularly among younger social media users (Ellison, Steinfield and Lampe 1164). Social network participation has shifted from special interest to everyday practice.

But as social networks have become commonplace, certain underlying principles necessary for their success have perhaps been taken for granted. Social networks rely heavily on the labour of their participants for sustained success and popularity, but this investment of time and effort goes by largely unnoticed or unchallenged by users of social media. The work required within a social network can be as small as clicking the 'like' button on a friend's Facebook update in order to display engagement and acknowledge approval, or as big as (in the case of Ravelry) producing an entire knitted garment, then cataloguing and sharing the details of this very tangible material object with an online social world. What distinguishes the labour of social networks from more "traditional" labour is that it is by and large immaterial and voluntary. Importantly, social network labour is interpreted as entertaining, fun or time-wasting, and this distinction from monetized work carries social meaning. The polarization of 'work' and 
'leisure' is so firmly established that it can be difficult to extract processes of labour from activities which are overall considered entertainment. Many scholars therefore employ the notion of affect in order to bridge this gap: Bratich and Brush (2007), Coté and Pybus (2007), Dowling et al. (2007) and Susanna Paasonen (2010) are but a few of many scholars who draw on affect in order to productively discuss online labour. In various ways, these scholars draw on the idea that the work that is being carried out in social networks carries interpersonal, emotional significance and is therefore meaningful. This, to some extent, justifies or masks the labour investment often necessary for successful social media participation. The work of the everyday social network participant may be opaque, but it must not be forgotten. Our labour investment is essential to the continuation of the social web, and how this labour is understood and carried out shapes our online environments. Drawing attention to the work we actually do seems both pertinent to and absent from wider discussions of social interaction in digital spaces.

Participants in social networks may not easily recognize their repetitive clicking, writing and reading tasks as 'work', but sites like Ravelry show that its users accept and impose an unquestionable element of 'hard slog', here in the form of manual labour, as necessary for reaping the benefits of social interaction and validation by their peers. It may seem like Ravelry demands a lot from its users by requiring a digitized component of tangible, material work in order to fully participate in the site's social space. However, it is unlikely Ravelers interpret acts of cataloguing and displaying as 'just' extra work. As Carolyn Wei has observed (2004, n. pag.), the process of establishing norms and behaviours within an online community is reflexive: structures and patterns of behaviour may initially be imposed through site architecture and codes of conduct, but the "actual practice" (Wei 2004, n. pag.) of the community's members will ultimately shape the direction and purpose of the community itself. This is crucial for understanding why Ravelry stands out: Ravelry's social network requires a lot of labour, but this is to a great extent work knitters themselves have determined to be important and meaningful.

The physical, repetitive act of knitting may not always be 'fun', but the motivation to persist and then share digitized mirrors of these efforts clearly serves a purpose. The meaning of manual work appears to expand when evidence of this work is 
catalogued and displayed digitally. The manual labour undertaken has already resulted in a material, knitted object which can be gifted, kept or sold independently of any connection to the online. But by translating this labour into digital records, the labour of knitting can also be used to build and maintain an online community. Ravelers can be understood to reap a triple benefit in this offline-to-online flow: the process of knitting itself is considered a pleasurable and for the most part relaxing activity, the material objects knitters produce have practical application, and the knitters' labour can be translated into digital texts which are used to strengthen ties within a social network. These ties are strengthened when Ravelers engage with and acknowledge each other's records of labour, which in turn encourages the creation of more material objects to digitize and validate. Ravelry is here by no means unique: validating other people's digitized records of manual activity can be found in any social network where members post about their daily lives, upload photos or otherwise create digital mirrors of their material, offline experience. ${ }^{2}$ What Ravelry brings to the table is an overt connection between offline labour and online validation.

What emerges from Ravelry's use of 'finished objects' as representing a particular user is an emphasis on offline, manual labour which in turn serves as an entry token into the social network. Self-representation happens at least in part through a physical object, the result of offline, manual labour which must then be "written into being" (Sundén 88) via text, photos, feedback from others and so on, in order for the self to be validated by the online community. This is valuable from the point of view of social media scholarship, as placing a material object between self and selfrepresentation is a dynamic infrequently encountered within social networks. Moreover, a finished object (for example, a sock) is not fully realized as a sock within the Ravelry community until it has been photographed and catalogued in its creator's notebook, perhaps to be viewed and commented upon by someone else. Evidence of creation must be present for successful participation in the network, and manual labour must be mirrored in a database entry (in itself another form of labour) in order to render itself visible for validation. This is the core aspect of the site which distinguishes it from other types of social networks - neither the finished object nor its creator are fully 'done' or realized until they have been shared with and confirmed by other Ravelers. This, in turn, is an important part of the labour process for the object's 
creator, which leads to the question: why is this validation of labour so important? How is labour on Ravelry made socially meaningful?

\section{What is Ravelry? A brief description}

The website Ravelry.com, established in 2007, describes itself as "a free site for knitters and crocheters." At first glance it appears to bear hallmarks of an apparently "typical" social network based on a niche interest, where people interested in knitting and crochet keep in touch and share resources through the site's vast array of options and features. After spending some time on the site, it becomes apparent that this overtly social aspect is but one of its many functions. Ravelry.com (from here on referred to simply as Ravelry) also hosts vast databases of shareable knowledge such as usercontributed knitting and crochet patterns, forums for tutorials, troubleshooting, showing off one's skills and socializing, and databases of materials such as yarn and knitting needles. Banner ads and links to online stockists of handicrafts supplies are also prevalent, and can be understood as a way for the site to gain advertising revenue in a manner less likely to irritate its members than the more conventional obstructive ads to unrelated sites ("Ravelry Advertising and Pricing" 1). Selective advertising also circumvents the need for members to pay for access while providing them with potentially useful information in their roles as knitters and crocheters, as all the advertising on the site is fibrecrafts-related. This reasoning is confirmed by the site's founder Jessica Forbes: rather than charging members, "we have tasteful and targeted ads for fibery products and companies that we as a community are actually interested in and want to support!" (Ravelry.com "About" September 2012).

On most social network sites, members represent themselves by providing a personal description that includes their likes, dislikes and interests on their profile page. What constitutes "personal information" varies from network to network, but recurring requirements are details such as name or username, age and location. Most also request a picture or avatar. A profile page will typically also demonstrate the member's social ties within the network in the form of a list of friends or contacts (boyd \& Ellison 213). The combination of these elements provides a snapshot of what is considered basic information about an individual, and profile pages are often fleshed out with details about the user's particular tastes and interests (cf. Liu 2007). Ravelry is 
no different from other social networks in that it is compulsory as part of the sign-up process for each member to set up a rudimentary profile page. Where Ravelry perhaps departs from "profile-centric" (boyd \& Ellison 216) social networks is that beyond this initial point, the Raveler's profile is rarely used as a nexus of interaction. Instead, Ravelers are represented through "My Notebook" - a collection of database entries recording and displaying the Raveler's 'finished objects' and material peripherals to these completed projects such as yarns, knitting needles etc. owned by the user. In fact, the prominence of "My Notebook" is such that the profile is often overlooked altogether. Jill Rettberg writes that on Ravelry, "each user has a 'notebook' (rather than a profile) where all his or her projects are displayed" [emphasis mine] (457). This means that when looking up other users on Ravelry, one finds much more information in their notebook than in their profile. In fact, if searching for users via Ravelry's own search engine, the resultant link will take you straight to the selected user's notebook, not their profile. The linking to notebooks rather than profiles as places where 'things happen' is a central aspect of both the site's architecture and normative dimensions. The Raveler thereby becomes represented in large part through his or her labour. Rather than simply writing about her- or himself in the manner of, for example, a Facebook status update, completing "My Notebook" with records of offline, manual production is necessary for gaining full entry into Ravelry's social world.

\section{History, membership and context}

At present, Ravelry has approximately 2,366,458 registered members distributed across the globe (Ravelry.com "statistics > users", 10 September 2012). The site has enjoyed steady growth in membership with 945,307 new sign-ups since 29 June 2011, speaking to its popularity and prominence as a fibrecrafts resource. The site itself is written exclusively in English, with English also being the dominant language in Ravelry's user-generated content, and it is this Anglophone Ravelry which will primarily be analysed here. There are some exceptions, for example in the patterns database, where it is not uncommon to encounter patterns in other languages, often with English translations available. Local handicrafts groups in non-Anglophone countries also have a presence on the site, but they appear vastly outnumbered by English-speaking ones according to a breakdown of groups by location (Ravelry.com/groups/browse/location, 10 September 2012). Ravelry does not otherwise provide its members or visitors with 
detailed breakdowns of membership statistics, so it is not possible to ascertain exact figures of Anglo- vs non-Anglophone members. Instead, the impression that this is primarily an English-speaking site emerges from the text, as finding content in other languages often requires refined searching.

The site was founded in 2007 by Jessica M. Forbes and Casey Forbes, known on Ravelry simply as Jessica or Mama Rav, and Casey a.k.a. Code Monkey (Ravelry.com "About"). According to Ravelry's 'About Us' page, the idea for the site came from Jessica, who wanted an easier way for knitters and crocheters to collect and share information online, and Ravelry was then built by Casey, a professional web developer and also Jessica's husband (Ravelry.com "About"). Ravelry employs a further two people, Sarah (no surname given) and Mary-Heather Cogar, whose titles are respectively listed as Community Support Specialist/Hostess with the Mostess and VP of Operations/DoGooder. Such a concise team of employees indicates that Ravelry is a relatively small undertaking commercially, despite its membership base of over 2.3 million. It should be noted that while 2.3 million may seem a high number, these are the individually registered members in total, not currently active users. While Ravelry does not publically provide a breakdown of membership statistics, it will display how many users are active at any given time, and how many have visited the site within the last 30 days. This information is provided after logging in, and its immediate presence emphasises the collective nature of the site. At the time of writing (7:34pm on Monday 10 September 2012 in Christchurch, New Zealand), there are 1788 Ravelers online, with 513,995 having been active on the site within the last 30 days (Ravelry.com "statistics/users").

While Ravelry's appeal is narrow, the site is by no means unique. As a specialized social network, it exists within a much wider network of crafts- and domesticity-related websites, blogs and social networks. Clear parallels can, for example, be drawn between Ravelry, craftster.org and etsy.com. Craftster follows a similar dynamic to Ravelry where users upload evidence of their crafts projects and tutorials for others for the network to comment on and validate, where etsy.com is a commercial site for buying and selling handcrafted goods with a focus on DIY handicrafts like fibrecrafts, metalwork, sewing and painting. ${ }^{3}$ A myriad of smaller online communities (typically blogs, LiveJournal.com 
communities, Facebook groups and so on) contribute to the presence of crafts and DIYoriented networks online.

\section{Social capital and Ravelry}

The idea that social capital is present within social networks, and therefore also visible as well as important on Ravelry, recurs throughout this project. The precise definition of social capital is difficult to pin down, as it is an "elastic" term with a number of interpretations and applications (Ellison, Steinfield and Lampe 1145). Despite this, the many differing definitions of social capital all speak to the productive potential of forming and maintaining relations with others within a social structure - a social network site being one type of such a social structure (Lee 716). As Lee points out, study of social capital within social media and Internet use has to date been inconsistent and yielded varying results (719), particularly when considering why people choose to form communities online. However, some consensus has been reached in determining that social capital "[is] linked to a variety of positive social outcomes" (Ellison, Steinfield and Lampe 1145), and is generally viewed as "a positive effect of interaction among participants in a social network (Helliwell \& Putnam qtd. in Ellison, Steinfield and Lampe 1145). This understanding of social capital as beneficial, perhaps even affective, plays an important role in attempting to answer the question of how labour is made meaningful on Ravelry. Bourdieu defined social capital as "a sum of the resources, actual or virtual, that accrues to an individual or group by virtue of processing a durable network" (qtd. in Lee 715), while Coleman points to social capital being a type of capital which "helps accomplish certain goals that may not be possible in its absence" (Lee 716). In other words, social capital is productive.

On Ravelry, social capital is textually accessible through the way members interact with each other using articulated and manoeuvrable links (often in the concrete form of hyperlinks) to other members. A Raveler who displays many connections to other members in her Notebook, profile, forum posts or other user-generated content displays a strong network, which can be drawn on for support, socializing, problemsolving and validation. As such, a Raveler can use her social capital - the productive potential of social connections to others within the network - to achieve social and personal goals. Building and sustaining social capital happens in large part due to the 
work and effort Ravelers put into their records of labour, as these records form nexuses of social interaction. Social capital is therefore difficult to separate from labour on Ravelry as records of labour tend to incorporate expressions of social connections as well. Evidence of both labour and social capital is, however, expressed within the multitude of texts that make up the site, particularly within Ravelry's alternative to the user profile, called "My Notebook". These areas or spaces will be approached as textual objects for the purpose of conducting an analysis of how labour is expressed and made socially meaningful within the Ravelry community.

\section{Ravelry's multiplicity of purpose: labour space, social space, marketplace}

The accrual of social capital on Ravelry allows members to reap benefits from their labour in multiple ways, and across most areas of the site. Although Ravelry's many functions often co-exist without overt linguistic or visual markers to distinguish them, it is possible to divide the site into three distinct spheres or layers. The edges of these layers overlap significantly, but at the core of each sphere is a distinct function or purpose which makes it possible to recognize and extract it from the content overall. It is perfectly possible to engage with a single sphere without venturing beyond it, but it is perhaps more common to move between them seamlessly, to the point where they appear intertwined. It is, for example, not necessarily immediately obvious to a Ravelry user that the site's social network functions and marketplace functions are not one and the same, as both permeate the site and appear co-dependent. Nor is it clear that records of personal labour exist independently from the site's social focus, as these records are put on the site to be seen and interacted with unless the individual Raveler specifically opts out of sharing with the network. In fact, making one's records of labour a platform for social activity happens by default. Again, the traditional user profile, established by boyd and Ellison as one of the hallmarks of a social network (211), is on Ravelry replaced by "My Notebook". If a Raveler wishes to opt out of sharing labour records with the community at large from his or her Notebook, this would require a change of settings and active monitoring/filtering of content.

As Ravelry is a highly detailed and textually rich object, it is necessary to utilize categorization as a way of clearly identifying the particular aspects of the site where meaning is created for and by its users. Devising a framework applicable to the site's 
content is therefore a useful tool for narrowing focus and identifying key sites of analysis. As stated above, the borders between Ravelry's spheres are diffuse, but distinguishable. In her 2009 conference paper "The economies within an online social network market: A case study of Ravelry", Sal Humphreys employs a similar "heuristic device" (240) in order to tease out separations between the site's many forms of transactions into distinguishable forms of economy for the purpose of analysis. In her research paper, Humphreys is interested in how Ravelry functions as a social network market, where the economies of gift-giving and commercial exchange overlap in a manner which challenges assumptions about their incompatibility. While Humphreys' focus is on economies, this project takes this idea of separating spaces as heuristic device further, by producing a taxonomy of all Ravelry's content. It is perhaps useful to think of Ravelry's spaces as open but interlinked, rather than layers or spheres, which give the impression of set boundaries. Employing the space metaphor, Ravelry can roughly be divided into three categories:

- Labour space

- Commodity space

- Social space

These categories form the framework for understanding the site. They have been chosen for their ability to represent the typical use of Ravelry by its members. Sal Humphreys' observations about micro-transactions and economies are encompassed within the commodity space, or marketplace, in this taxonomy. However, this categorization potentially excludes ways of using the site not visible in the text - it does not, for example, account for the behaviour of active but non-contributing members, the 'listeners' (Crawford 2009) on the site as this falls outside the scope of textual analysis. ${ }^{4}$ These 'listeners' are still addressed within the site architecture, for example by tracking page view statistics, and they are interpellated in the same manner as more active or visible users. The invisible Raveler is therefore always a potential visible Raveler. It seems reasonable to assume that what attracts listeners is not too different from what attracts visible members. There may be other ways of interacting with the site unaccounted for here due primarily to obscurity; it is difficult to locate and account for those who do not wish to be found. While these categories are broad enough to encompass typical use of the site, they are not catch-alls. Instead, they provide a way 
into an in-depth analysis of those most common and prominent activities and functions that attract and retain members.

\section{"My Notebook"}

It is important to select an approach to analysis which provides access to the parts of Ravelry where meaning, as relevant to the research questions, is found. This means it is necessary to look at the parts of the site where labour is displayed, as well as the parts where labour is discussed and otherwise interacted with, and where social capital is accumulated. How is labour expressed, and how is it talked about? As answers to both these questions reside within and are accessible from the object, conducting a textual analysis seems most productive. This will take place against a backdrop of existing theory relating predominantly to social networks, labour and "new" domesticity. The analysis will attempt to address and incorporate the multimedia nature of the site: content recording, cataloguing and displaying labour is as likely to be found within visual imagery, or particularities of social media technologies (such as the ability to +1 or add to favourites) as within the written word. Producing a taxonomy of this multimedia content, which reaches beyond simply writing, will therefore form a starting point and foundation for an extension of web-based textual analysis.

The role of "My Notebook" as a significant aspect of labour space is emphasised by where it is placed on the Ravelry website. Immediately after logging in, one is taken to a welcome or start page featuring an RSS feed of community news and events, and it is possible to navigate straight from this page to most of Ravelry's content. Features customizable by the user are positioned in a horizontal strip above the RSS feed with "My Notebook" as the first link, right-aligned within the horizontal strip, right next to the Ravelry logo. This places the link to "My Notebook" in a privileged position in terms of conventional Anglophone page design - when reading from top to bottom, left to right, "My Notebook" is the first hyperlink which navigates away from the start page.

The purpose of "My Notebook" is not entirely clear through its name alone. The sub-menu, which appears when hovering the cursor over the "My Notebook" link, illuminates its purpose slightly better: "projects, stash, queue, favorites, friends, groups \& events, needles \& hooks, library, message box, blog posts, contributions, purchases" are all individually clickable links within this menu and indicate that this is where the 
Raveler can customize his or her own presence on the site. "My Notebook" is therefore individual Ravelers' space for creating records of what they have made, where they have interacted and what they own. Visually, "My Notebook" follows a clear structure of fixed links to the categories described above inside a narrow column or text box on the right side of the page, with details of the Raveler's own contributions to each section taking up most space on the page next to the fixed links. "My Notebook" also contains advertising in the form of a clickable image (banner ad) underneath the text box of fixed links. The presence of advertising remains constant, but new ads are displayed upon refreshing or navigating to a different page within "My Notebook".

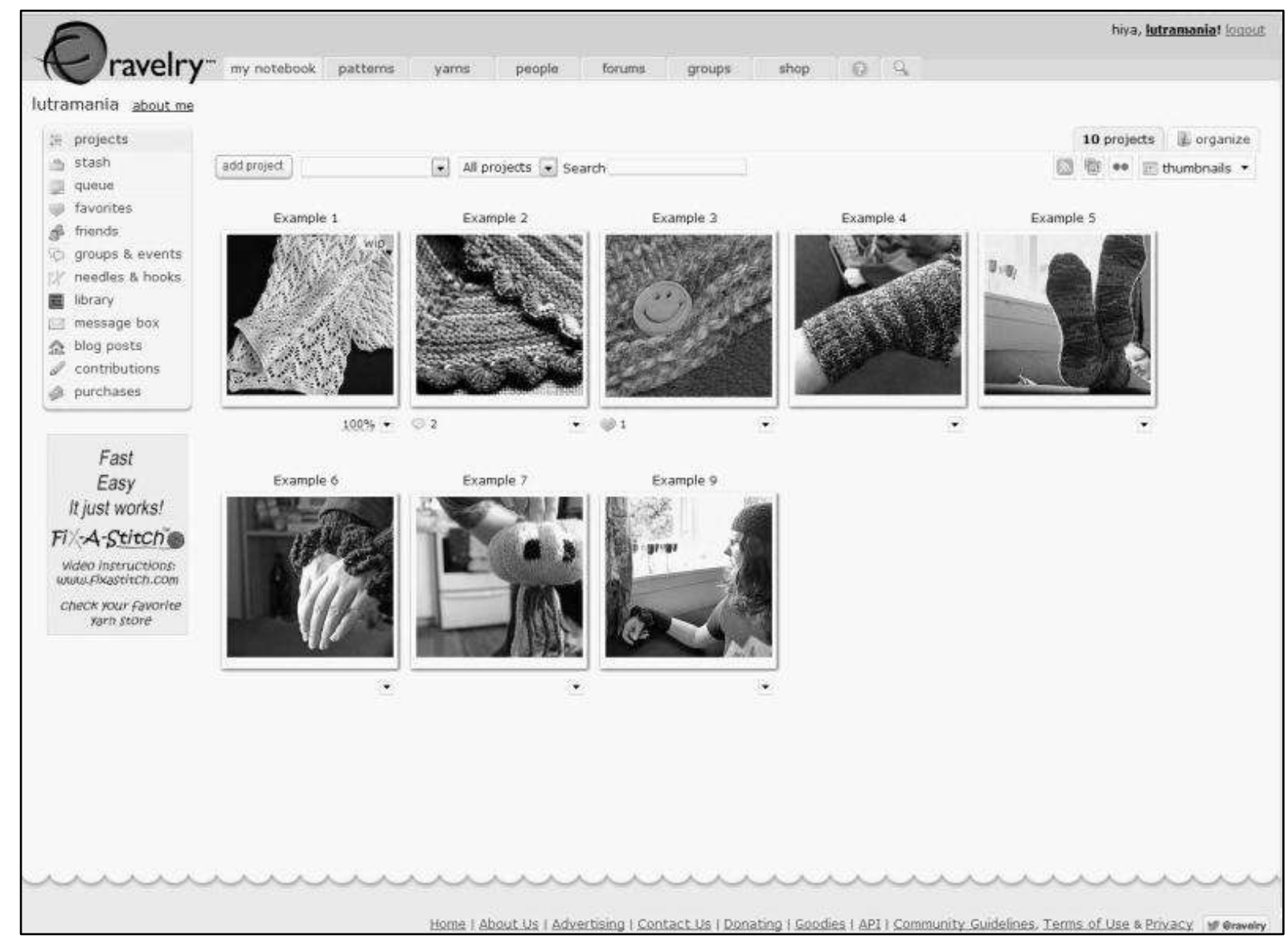

Figure 1 - "My Notebook"

In order to better understand how "My Notebook" works as a replacement for a profile page, it is necessary to explain its core features. Here, they are divided into categories based on which 'space' they are most closely aligned with. This classification is not definitive, but demonstrates which type of space is most apparent in each entry elements of all types of space are found in each function, as indicated in the descriptions. 


\section{Labour space}

This is where records of offline, manual labour are typically stored, and also where the necessary information for current and future labour can be found. It is possible to engage with Ravelry purely through its labour space; it is not necessary to participate in the social network or utilize the marketplace in order to access, for example, patterns. Sub-menu items associated with this space include:

- "Projects": where any individual knitting or crochet project the user is currently working on or has completed in the past can be recorded and displayed. Typically, a photo of the 'finished object' or 'work in progress' is displayed along with details about materials and pattern used, completion time and problems encountered. All projects are visible to all Ravelers by default - one must specify otherwise in one's account privacy settings if desired.

- "Queue": this is related to the "Projects" tab as it displays any projects the Raveler has acquired a pattern or idea for, but has yet to commence. Once an item in the queue is started (and updated on the site), it moves into the "Projects" tab.

- "Contributions": potentially an expression of both labour and social space, Ravelers can here contribute to the site's depository of knowledge (encouraging and facilitating future labour) by uploading patterns they have written, or details of yarn they have spun and/or dyed. In the case of patterns, they can restrict the dissemination of their shared knowledge by making the pattern available for purchase only. The "Contributions" tab will store records of any pattern or yarn the Raveler has shared with the site. It is also possible to upload records of patterns and yarns found elsewhere, provided licencing allows and they do not already exist in the database.

\section{Commodity space}

Commodity space encompasses the site's built-in marketplace as well as areas where ownership of commodities is recorded and displayed. It is intertwined with the social; the incorporation of systematic displays of possessions and commercial transactions with social network functions suggests this display is socially meaningful. However, it stands as a separate category as the site incorporates many social network 
functions which are not fundamentally commercial, plus the site serves as the business platform for many small-scale vendors. Areas of the site which fall under this commercial heading include:

- "Stash": this is where Ravelers create records of any yarns they currently own or have used in the past, and they can indicate whether their current yarns are available for sale, trade or gifting. Yarn info such as brand, range, colour and weight is mostly gathered from a pre-existing database; it is not necessary to input all data manually although this can be done if required.

- "Needles \& hooks": a simple spreadsheet-like page for recording knitting needles and crochet hooks in one's possession. These can be cross-referenced with patterns for compatibility.

- "Purchases": records of purchases initiated directly through Ravelry are autogenerated and stored here. These records show details such as when the purchase took place, who received money and how much was paid, as well as a title of the item purchased. If the item purchased was a download, the downloaded file can be accessed here.

- "Library": a reference management system used to keep inventory of relevant written works such as books and magazines in the Raveler's possession, as well as hyperlinks to online material and stored PDF files.

\section{Social space}

These are the spaces where Ravelers interact directly with each other either in the form of written correspondence such as messages or through types of interaction such as 'friending' other Ravelers or adding projects, patterns etc. to one's favourites. Social space also encompasses displays of belonging by showing one's connections to the site's social world in the form of friends lists and group memberships. Social space on Ravelry includes:

- "Favorites": Almost anything on Ravelry can be added to the scrapbook-like "Favorites" page by clicking a heart-shaped favourites button. This includes projects, patterns, designers, shops, yarns and yarn brands, stashes, tags, forum posts or 'clipped ads' - adverts found around the site and added to one's favourites. 
- "Friends": the list of social connections in the form of people 'friended' other social networks typically place on a user's profile page.

- "Groups \& events": links to any handicrafts group with a presence on Ravelry the user belongs to, as well as events (on- or offline) organized by these groups.

- "Message box": the inbox for the site's in-house messaging system.

- "Blog posts": Ravelers have the option of displaying a feed of their off-site blogs to their profile here via RSS (Really Simple Syndication).

As this brief taxonomy of content demonstrates, "My Notebook" incorporates three different arenas of activity. It is difficult to determine whether one type of space is genuinely dominant; arguably the focus on records of labour as representative of the individual speaks to labour space as a prime purpose, but these records are made meaningful through their incorporation with commodity and social spaces. Social space permeates most features of "My Notebook" through other users' ability to share, comment, direct message and add to favourites, whereas commodity space is always going to be visible within labour space with both advertising and more surreptitious links to vendors and retailers being integrated with, for example, a completed record within the "Projects" tab.

The spatial metaphor has been chosen for its connection to materiality. Reminders of the offline, material world are always present on Ravelry, and employing a material metaphor therefore adds a degree of consistency to this taxonomy. While these spaces overlap (as demonstrated for example by "Contributions", classified as labour space but encompassing social space if desired by the Raveler) they can also exist independently. If desired, a Raveler can choose to only engage with one particular type of space. It is fully possible (and probably quite normal) to, for example, only use Ravelry as a patterns database, never interacting with others or using the site for financial transactions.

\section{A little background: methods for studying online environments}

In terms of method, textual analysis is perhaps not the most common approach to studying websites and social networks. In contrast to much existing research in the field, it does not allow researchers to directly seek out the motivations, feelings and thoughts of the website's users, nor does it address the offline, worldly contexts of their 
media use. There exists a wealth of ethnographic work on new media, its users and technocultures, which will be drawn upon for this study, but it is important to highlight this methodological point of difference. There has, perhaps, existed an investigative drive within new media studies which often takes research in the direction of ethnographic participant study. This is clearly illustrated in Virtual Methods: Issues in Social Research on the Internet (Hine 2005), where social research online is implicitly equated with ethnographic research: nine of the book's fourteen chapters speak directly to issues with, and provide solutions within, ethnographic methods. The types of ethnographic work Virtual Methods draws on can be particularly insightful, not only for their individual merits, but for the overall impression of where and how meaning was thought to be accessible within earlier technocultural studies. In other words, the need to ask participants for their motives within these (at the time) relatively unchartered territories highlights that which is not studied - the websites, MUDs, online games and other interactive arenas as textual media objects in their own right.

There are times where an ethnographic approach could be supplemented with textual analysis in order to more clearly articulate its argument. For example, M. Sveningson's chapter “Cyberlove: Creating Romantic Relationships on the Net” in the oft-cited work Digital Borderlands (Fornäs et al, 2002) utilizes strictly ethnographic methods (participant interviews in particular) in order to explore the role of chat rooms in building romantic relationships. But it has been noted that this approach does not fully explore online romance and dating from a social research perspective, as "ideological constructs like "love" and "romance" are not interrogated", and this omission seems related to method rather than theory (Bury 2004). By contrast, Jenny Sundén's chapter in the same book, “Cyberbodies: Writing Gender in Digital SelfPresentations" combines textual analysis with participant interaction in MUDs 5 as a way of adapting to an online setting where accessing meaning requires this adaptive approach. Sundén interacts with participants as an ethnographer observing and interviewing, but she also conducts a textual analysis, describing the appearance and content of the virtual spaces she encounters and deriving meaning from these spaces themselves. Throughout “Cyberbodies”, Jenny Sundén marries these observations about online space to participant observation and discussion. This mixed method approach illuminates the significance of the textual. 
Another type of method is the purely quantitative or statistical, where data is collected, for example from websites, using purpose-built software or other established tools. Within the Humanities as well as Computer Science, this type of research is often utilized in the study of social networks, particularly as a way of mapping user behaviour and relationships, or even to study the behaviour of social network technologies themselves, like in Finin et al's Social Networking on the Semantic Web (2005). Typically, quantitative data will be combined with social network theory, as in C.

Haythornthwaite's "Social networks and Internet connectivity effects" (2005). Here, she assesses email behaviour among academics and distance-learners as a way of exploring the "impact of communication media and the Internet on connectivity between people" (125). Haythornthwaite utilizes existing work on strong and weak social ties within social networks as her theoretical foundation, and conducts a study aimed specifically at the use of social media within education and its "implications for planning and policy relating to choices regarding communication structures and information dissemination, and future uses of the Internet" (142). As with the studies discussed previously, the medium in question is treated as a motivation for studying the social behaviours surrounding it.

\section{Andrea Press and Sonia Livingstone (Questions of Method in Cultural Studies} 2006) noted that there has perhaps been an over-privileging of ethnographic methods as a "default setting" within Internet studies where the content which arises from the technology itself is not the focal point, and that striking a balance between media-asobject and media-as-text is desirable in order to fill certain gaps in the field. It should be noted that Press and Livingstone were responding to what is now considered early work within new media studies, and much innovation in method has since taken place. For example, scholars like Tiziana Terranova, Lawrence Lessig and Geert Lovink have done much work to address the political economies which shape and influence new media production, content and usage. This is another approach which provides further insight into aspects of new media and its uses which may otherwise be quite opaque, and a political economy approach often necessitates a meta-analysis of content as well as theory. Overall, there exists a wealth of methods for studying new media, but many of these methodologies have only been applied relatively recently. These methodological developments will be addressed in more detail in chapter two's literature review. 
The selection of secondary material should also be mentioned here, as supplementing scholarly theory with works from popular culture will at times form part of the method. The need for this has become particularly evident within the social histories of handicrafts and domestic labour, particularly as they relate to "new domesticity" and new media. Sites like craftster.org and Esty.com have already been mentioned, and such tie-ins to handicrafts and domesticity from popular culture form part of the foundations for this project. Academic work will form the primary theoretical foundations for this project, while popular literature and other works such as blogs and websites will typically be addressed and analysed as media texts. Given the relative lack of material relating labour to social media and domesticity, in cases of gaps within academic work alternative literature will also be evaluated and addressed.

\section{Understanding writing, knitting and interfacing through textual analysis}

Jenny Sundén’s study “Cyberbodies: Writing Gender in Digital Self-Presentations” has to an extent inspired this thesis. Based on her PhD field work and dissertation, her chapter in Digital Borderlands analyses the way gender is performed purely in written form within online environments known as MUDs or Multi-User Dungeons/Domains. Sundén argues that these environments are best understood as textual (90), and draws attention to the importance of interfacing, or the process of mediating between the "embodied self" (80) and the self written for and to the social network. She calls the interface, or the technology used to interact with the social network, "a crucial site, a significantly ambiguous borderland between human and technology" (80) while the MUD itself is a kind of textual space. Sundén's observations break with the now discredited ideas of the "virtual community" as sites of unrestrained identity-creation (Lovink ix), where the construction of a digital self was thought to be unimpeded by existing social structures, norms and expectations.

Yet, Sundén observes that in an environment where there is no verifiability of coherence between subject and textual representation of self, users still prefer to write themselves in ways that resonate with their offline social realities, if not mirror them. She addresses this in terms of gender, and finds that most users prefer to write themselves in gendered form within the MUD although this is not necessary (107). Additionally, most prefer to write themselves in accord with their gender identity 'in 
real life'. And interestingly, most prefer to write themselves as slightly flawed, particularly in their performance of gender, as personas without imperfections are met with often have difficulties starting conversations and accessing the MUD's social world $(80,107)$. Her findings come primarily from text found within MUDs, but she also played the part of ethnographic field worker by participating in the MUDs herself, identified as a researcher (89). While she engages directly with MUD participants, their responses are themselves treated as texts that form part of, and cohere with, the bigger object. The parallel to Ravelry is therefore twofold: firstly, Sundén addresses the online environment as text, and secondly, she realizes there is a thing that stands between the self and the online world, negotiated through the process of interfacing. In the case of "Cyberbodies", this thing is writing. Ravelry adds new layers to this as it requires not only a thing in the form of writing, but also imagery and, most importantly, an actual physical object - literally a thing - for successful participation in the social world. Placing a physical object between self and social network generates something of a conundrum, as the "thingness" or physical existence of the object is only verifiable through its digital representation.

Textual analysis has therefore been selected for its ability to access meaning within the site as text. Another benefit of approaching Ravelry as text is the immediate availability of material - what is visible is what is studied. This means access is straightforward, and claims about content are verifiable by any third party. More significantly, it gives the researcher the same ability to interact with the text as members of the site; we see what they see. The methodological implication of this is that the researcher has direct access to the same sites of meaning as the Ravelry community in general. The researcher can see the flows of interaction and movements between the previously outlined spaces, so analytical tools can be applied directly to these sites of meaning without the need for interaction with participants. Ravelry is also textually rich: the wealth of material is as promising as it is overwhelming. As with the Internet in general, the site's perpetually fluctuating content challenges any method employed. While most content remains available permanently within Ravelry.com, permalinks ${ }^{6}$ may not always be possible, and in cases where displayed images are selected randomly from a pool, the same page may not look the same to two users accessing it at different times. These problems will be addressed where necessary, but should not be significant 
as they are sporadic and make up only a small proportion of the site's content. In other instances, the changing nature of the content is in itself a site of meaning and will therefore be incorporated into the analysis.

\section{Thesis structure and chapter outlines}

Each chapter in this project is devoted to a particular core idea or concept, centred on Ravelry's spaces of interaction discussed above. Chapter 2 addresses the need for critical assessment of labour within social media in order to understand its significance, and investigates the scope and content of existing scholarly work on labour within online environments. This chapter also conducts a review of important theory within social media research in general. Recurring concepts encountered within new media research, such as immateriality and affect (see Lazzarato 1996, Hardt and Negri $2000,2004)$, will be interrogated with the aim of establishing whether or not they adequately account for the dynamics observed on Ravelry. Based on this review of theory, Ravelry's points of difference as well as its adherence to social media norms will be established. This sets the stage for a more in-depth analysis, particularly of Ravelry's status as a specialized, niche social network. Chapter 3 places Ravelry as a niche network belonging within a wider context of hip domesticity in which the homemade, crafty and otherwise DIY has gained traction as an arena for online interaction where domestic labour is socially meaningful. While chapter 3 will focus on the social, affective aspects of this hip domesticity, chapter 4 looks at the significance of handicrafts and DIY as popular commodity. The socially meaningful world of handicrafts does not exist outside a commercial realm, as demonstrated through Ravelry's intertwining of social, commercial and labour-oriented spaces. In broad strokes, chapter 2 can therefore be read as an investigation of labour space, chapter 3 looks at social space and chapter 4 contributes an analysis of commodity space.

Chapter 2 will encompass a literature review with particular focus on the labour of new media, or so-called immaterial labour. How is the labour of social networks currently understood, and what gaps may need filling? Maurizio Lazzarato's idea of immaterial labour (1996) is evaluated extensively in this chapter as it forms the foundation for so much ensuing scholarly work on labour within ICTs and social networks. The success of a social network requires an implicit agreement between its 
users that they will all contribute and 'do work' by creating some degree of content while interacting with the content created by others - both being examples of what is currently typically described as immaterial labour. Ravelry adds another dimension to this by requiring material labour for successful participation, then digitizing this labour and making it the focal point of interaction. By making labour visible and important, Ravelry calls the immaterial and disembodied into question. Based on the literature review, there is room here for making a contribution to the way labour is understood as significant to the dynamics of a social network. By introducing a concrete, physical object, the online and 'immaterial' is firmly anchored to the offline and worldly, contradicting notions of disembodiment.

Chapter 3 engages primarily with the concepts of hip domesticity and affective labour. Firstly, the term 'new domesticity' will be explained and evaluated, with 'hip domesticity' suggested as a more current manifestation of domesticity, particularly online. Hip domesticity's relationship with affective labour will then be addressed, drawing on Hardt and Negri's efforts to flesh out the notion of affect $(2000,2004)$ which they base on Lazzarato's famous work on immateriality (1996). Affect is defined here as a subjective or emotional quality which renders labour socially meaningful. It is related to immaterial labour, but exists outside the immaterial as demonstrated, for example, by how the very tangible, physical work of knitting is imbued with affective meanings. Affect is often also specifically talked about in relation to gender, particularly the affective notions which surround 'women's work' such as domestic labour (cf. Hardt and Negri 2000, Cowan 1983). A few examples within the broad range of domesticityoriented websites will be analysed in order to illuminate this relationship between affect, labour and gender. A type of gender performance appears to coincide textually with hip domesticity across a range of websites. Ravelry ties this gender performance together with affect through the way this intangible, affective quality is vital to the way labour is recorded and displayed, against a backdrop where one's gendered social reality offline is carried into the online. These ideas will be discussed in relation to social histories of domestic labour and the making-visible of 'women's work', which have led to current understandings of new (or hip) domesticity.

Chapter 4 discusses the relationship between commodity, commerce, selfrepresentation and validation on Ravelry. It will fill the 'commercial gap' not previously 
addressed when discussing immateriality and affect by speaking to the more businesslike, monetized dimension of labour and consumption on the site, drawing on recognizable structures of domesticity-related commodity consumption found throughout popular culture. Incorporating the commercial adds complexity to the notion of affect, as affective objects and affective labour form part of Ravelry's marketplace, but is simultaneously viewed as a contradiction of consumer culture. It is clear from the prevalence of advertising, business transactions and other types of commerce within the text that commerce and commodity both play part in Ravelry's social dynamics: does this influence the social capital gained through records of labour? In other words, does consuming the 'right' commodities increase a Raveler's social standing? If so, there is an overt connection between commodity consumption and social meaning. This problematizes the idea that Ravelry's overarching purpose is purely affective, and aligns the site with a more conventional pattern of commodity consumption.

Investigating these three main dimensions - labour, affect and commodity allows for a thorough analysis of Ravelry. This investigation illuminates the social meaning of labour within an online social network. Ravelry illustrates the importance of doing work in order to maintain strong social ties online. Unlike many other social networks, this work does not purely take place through the interface of a computer or other internet-enabled device. It is necessary to place a tangible, physical object between self and social network, which bears witness to a flow between the off- and online not often explored within new media literature. This project aims to illuminate this gap, offering the case study of Ravelry as one way of addressing the meaning and importance of labour within social networks. 


\section{Chapter 2 - Finding the work in social network: an overview of social media labour}

\section{Chapter overview}

The work that we do as social media users is intrinsic to the way social networks function. Consistent input of labour from participants shapes the content of social networks. This labour may range from the "thinking and clicking" tasks involved when interacting with a social network through a computer to doing manual work offline in order to later digitize and share this labour with a social network. Ravelry combines these online and offline processes in a manner that renders this labour visible. Thinking of social media participation in terms of doing work may seem a strange proposition. However, as Ravelry demonstrates, labour and doing work can contribute to a social network experience, as expressed through Ravelry's labour space. The purpose of this chapter is to form a foundation for understanding the significance of labour within social networks.

A range of scholarly work has been published in recent years on the types of work required by and for new media - from the technical, highly skilled work of writing code and publishing web pages, to the mundane, everyday clicking and writing tasks required for interacting with friends in a social network, with numerous examples of other types of labour in-between. Ryan Bigge ("The cost of (anti)-social networks" 2005 expresses surprise at the "amount of work" (n. pag.) this routine of participating in an online community entails, thereby opening up a debate about the relationship between labour and social network. Central to this chapter is a review of literature published in this particular scholarly 'neighbourhood'. This chapter asks: what are the current approaches to thinking about the work of belonging to social networks? Can these efforts of belonging be thought of as work at all, and how is labour a relevant concept here? Existing literature on the topic of social networks suggests that there is a gap in scholarly attention to the notion of work. How is this labour being talked about in existing literature? What concepts and explanations are employed, and are they adequate for describing the breadth and nuance of all the types of labour involved in social media participation? How and where labour is (or perhaps more tellingly, isn't) discussed and where there may be gaps in existing literature will then form a 
foundation for an analysis of the work social media users need to undertake in order to participate successfully in social networks. This labour analysis will then be applied to Ravelry.com's labour space as an example of an online community where these ties to labour are easily recognizable and explicit.

Before commencing with this combined literature review and labour analysis, it is therefore important to establish where and why labour fits as a relevant concept. Understanding the significance of 'work' within social media is therefore beneficial. Labour is an extremely broad concept, spanning everything from invisible, mental tasks known only by the subject, to overt physical labour interacting with and altering the world we live in. The success of a social network hinges on the 'work' of its users - the activities and engagements necessary for online presence - yet all this labour goes by largely unquestioned by participants and observers alike. Why, given this, should we think of social media participation as work? What kind of work is it? Labour in the context of social networks is called into question because of both its ubiquity and its invisibility. There appears to be a blind-spot here, which is evident in existing scholarly work: exploration and analysis of labour amongst social media participants certainly exists, but scholarly writing on the subject is fractured and scattered, making it difficult to predict where such debates will crop up. More scholarly work appears to have been put into the analysis of labour among those who produce new media technologies than those who use new media technologies. But the work of the average social media user, the work that forms the backbone of the social network by constantly generating new content and strengthening existing social ties, is poorly accounted for. Problematically, the terminology used to explain social media labour is also often inconsistent. This chapter will account for some of these inconsistencies and discuss whether or not it is possible to pin down broad, oft-employed terms such as "immaterial" and "affective" labour with more concise, easily applicable definitions grounded in specific social network sites.

\section{The new media worker}

There has been a slight privileging of the work of the specialist (programmers, web designers, graphic designers and other creators of specialized content) within writing on new- and social media labour. The work of the non-specialist user does not 
begin to significantly appear in literature until around the year 2007. Earlier writing, such as that by Terranova (2000), Gajjala and Mamidipuni (2002), Gill (2002), and Poster (2004) highlights the specialized professional or professional technological environment (such as an office or an online business) as the site of new media labour. Tiziana Terranova and Mark Poster both move beyond this in their key works "Free labor: Producing culture for the digital economy" (2000) and "Workers as cyborgs: labor and networked computers" (2004) respectively, allowing for some inclusion of, or blurring of lines between the new media creator and the new media participant. However, this distinction between early literature's relative blindness to participant labour and more recent literature's eagerness to acknowledge it becomes clearer in later work, such as that by Coté and Pybus (2007), Paasonen (2010), Bratich (2010) and Martens (2011). The shift in focus from the monetized labourer to the private, amateur user is evident in the choice of language used to describe the different types of worker: where Terranova speaks of "free labor" [sic], Paasonen speaks of "labors of love"; Mark Poster speaks of workers as cyborgs while Martens addresses the "transmedia teen" engaged in immaterial labour, unaware of or unconnected to the programmers and developers who make their "immaterial" work possible. Confusingly, immaterial labour also encompasses the labour of the new media worker, as defined by Maurizio Lazzarato in Immaterial Labour (1996). This poses some linguistic challenges, as the same concept appears to be utilized to talk about two different things. This chapter will unpack this notion of immateriality, and suggest that in the context of social networks like Ravelry, the 'immaterial' labour of its participants can be better understood as a process of interfacing (Lazzarato 1996) than a stand-alone type of work unconnected to materiality.

The notion of immateriality recurs in the writings cited above, as indeed it does in practically all scholarly work published on work, labour, social media and ICTs. There appear to be two separate and quite specific ways in which new media labour is discussed but, confusingly, these discussions utilize the same or highly similar terminology. The very broad concept of immaterial labour, defined by Hardt and Negri as the labour involved in "production of services [resulting in] no material and durable good" (293) has been utilized to describe a range of labour phenomena online. The first category (appearing as early as 1997) is often referred to as "new media work" or 
“digital labour" (Terranova 2000). New media work encompasses activities such as conceptualizing, designing, programming, publishing and maintaining web pages and social media, as well as all the other technical work necessary for new media products to exist and function. The people who undertake this work then become new media workers or "digital workers" (Terranova 39). This is perhaps the most straight-forward, orthodox understanding of what labour within new media means: new media work is the labour undertaken by specialist workers who produce and maintain the technology non-specialists can interact with. This encompasses a great degree of creativity and artistic expression which is still highly specialized; new media work is not all about the 'hard code' but requires many other artistic components and offers diversity in skills and careers (Gill 43). Geert Lovink, for example, speaks extensively of artistic labour within new media in Zero Comments: Blogging and Critical Internet Culture (2008). Here, new media is viewed as a platform for artistic endeavours, and Lovink critiques the dynamics of this creative, collaborative type of labour.

What both "hard code"-based and more ephemeral, artistic approaches to new media labour have in common is a high degree of technical knowledge as prerequisite, and workers in both (typically overlapping) areas are specialized new media workers. The working conditions of these "Web-workers" (Gill 10) have been subject to extensive analysis in which observations about intense, deadline-driven, exploitative and uncertain employment practices recur, mitigated in the minds of new media workers as well as wider society by flexible working conditions, perceived egalitarianism, the ability to freelance and the "cool-factor" of working with cutting edge technology (Gill 2002, Gill 2007, Gregg 2008). New media work is here understood as a subset of socalled immaterial production which includes "audiovisual production, advertising, fashion, the production of software, photography, cultural activities, and so forth" (Lazzarato $1996 \mathrm{n}$. pag.). This classification is both broad and vague - the common thread here is creativity and creative processes resulting in the professional creation and distribution of commodities, but the different types of work required make it difficult to draw a clear line between the manual and the immaterial. For example, both computer programming and photography contain a very tangible aspect of manual labour: while a creative process lies at the heart of each project, both require a degree of monotonous repetition in order to arrive at the best possible result. Often, this labour is 
undertaken on a contractual basis not as a creative outlet for the photographer or programmer, but to meet the needs of a client, thereby creating commodities.

While there are undoubtedly many pitfalls associated with both this classification of labour and this type of new media work (as shown particularly through Rosalind Gill's work), the focus of analysis here is not on the disadvantages of being a new media worker. What is of interest is how this type of labour is classified and categorized as its own entity, quite separate from the labour of participation by the users of new media. Discussions of the new media worker demonstrate the beginning of a wider academic debate regarding the sheer amount of work that is necessary to sustain digital content, from both creators and the users of these technologies. The early debates may have been centred on the professionals who create the technologies we interact with, but later debates have branched out into all the other types of labour necessary for the survival of ICTs. Most of the texts cited above were published prior to boyd and Ellison's oft-cited definition of social networks as "web-based services that allow... a public or semi-public profile within a bounded system" where individuals "articulate a list of other users with whom they share a connection" and where users can also "view and traverse [these lists] of connections" ("Social Network Sites: Definition, History, and Scholarship" 211), as it was around this time that serious attention began to be paid to the users, rather than the creators, of social media. The work of these users, the labour of participants, forms the second category of work within new media. New media labour has been the term chosen to describe the work of IT professionals and other creators of online structures that bring social networks into being. The second category encompasses the labour of the end-user or social media participant. Unlike new media work, this is not professional, monetized labour. Instead, it is the tasks undertaken for the purpose of participating in a social network. Immaterial labour or affective labour are concepts used as catch-alls for this type of work, as seen, for example, in Bratich's “The digital touch: Craft-work as immaterial labour" (2010), Paasonen's "Labors of love: netporn, Web 2.0 and the meanings of amateurism" (2010) as well as Martens' "Transmedia teens: Affect, immaterial labour and user-generated content" and Coté and Pybus' "Learning to immaterial labour" (2007). Here, a blind-spot has become apparent as the labour of the consumer or participant appears not to have any concrete term or definition of its own. 'Immaterial 
labour' is borrowed from Lazzarato, Hardt and Negri and others, but what it describes in the context of social media participant labour only covers a small aspect of the actual labour carried out, as will be demonstrated in the analysis of Ravelry.

\section{Contextualizing immaterial labour: a brief history}

Immaterial labour, a term attributed to the Italian sociologist Maurizio Lazzarato (1996, n. pag.), is by no means specific to new media, but has been utilized to describe the types of labour not easily explained through more orthodox understandings of the concept of work. Lazzarato defines immaterial labour as "the labor that produces the informational and cultural content of the commodity" (134), and argues that immaterial labour accounts for new types of work brought about in post-Fordist business models where workers must act as "interfaces" between creativity, technology and commodity production (1996, n. pag.). In Lazzarato's definition, immaterial labour encompasses the knowledge-based or intellectual work necessary for production in industries where the production itself is largely mechanized and requires little manual labour. Yet, creativity, "informativity" (Lazzarato 1996, n. pag.) and cultural relevance need to be present in the commodity. Immaterial labour, then, is "invisible" in the sense that it is a creative process occurring largely within the mind of the worker, and the worker must then communicate with another link in the chain of production (typically this means interacting directly with a machine) in order for the creative process to be carried out, leading to the creation of a commodity. An "immaterial labourer" is responsible for the creative process spawning an idea for a product, and then communicating this idea either to other professionals or directly to technology. This is what Lazzarato calls "interfacing", and he argues that this type of mindwork permeates the modern workplace. A large part of a worker's day is spent on labour which is intangible until successful interfacing has taken place, yet workers are often expected to undertake this immaterial labour in a similar manner to working on a production line. Graphic designers, for example, are often used to exemplify the immaterial labourer as they must come up with an idea and bring this idea to life by interfacing with technology, for example by creating their designs entirely on a computer. This is sometimes thought of as a creative process "made to order", as graphic designers typically work to create commodities which must satisfy the demands of a client. This understanding veers back towards the new media worker and the monetized aspects of "immaterial" work, and 
doesn't seem to fully cover the labour investment of, for example, the average Facebook user. The process of interfacing, however, must occur in order to participate in a social network.

So how can it be that immateriality has become such a prevalent concept for discussing labour in online settings? By Lazzarato's definition above, there seems less scope for including non-monetized, voluntary or "affective" work. Lazzarato's theory describes both mental and physical labour within the context of commodity production as immaterial. Hardt and Negri's definition of immaterial labour as something occurring within the "service sector at the top of the information economy" (293) broadens its scope, but still relates it to industrial production within a knowledge economy. Yet, the term 'immaterial' is also commonly used to describe labour which is creative or affective outside commodity production, as illustrated through, for example, Martens' “Transmedia teens: Affect, immaterial labour and user-generated content" (2011). Immaterial labour appeals as a concept because Lazzarato leaves a broad scope for interpreting the term within the framework of new media; Hardt and Negri open it even further. As mentioned above, within scholarship the term is often used in two distinctive ways: either to describe the tasks of a new media worker, or to explain behaviours and actions of online community participants. This actually appears consistent with Lazzarato's own text, where the term is used in a similarly divided fashion. Lazzarato speaks not only of the mind-actions of creating and designing, but also of the very physical acts necessary to interface with some form of technology, for example via typing. Should these quite different processes really be grouped together under the single header of immaterial labour? The Facebook user must translate the 'mindwork' they are undertaking into physical interaction with an internet-enabled device in order to be part of their social reality online. Where is the immateriality in touching a keyboard, or sliding one's finger across a smartphone screen? In the case of Ravelry users, where is the immateriality in the fuzzy object resulting from a lengthy, manual labour process? Here, interfacing seems a more pertinent term.

The development towards including the social media participant in discussions of labour has brought about the need for a refinement of terminology, separating the participant or the user from the monetized Internet-worker. It is worth noting that it is common for one person to occupy both roles as both producer and consumer, and this 
'prosumer' (Toffler 1980, Tapscott 1995)7 continues to be the focus of much academic attention (cf. Ritzer and Jurgenson 2010, Bianco 2009). The second category, the work of the social media user as participant, does not currently have a term of its own, but falls under the umbrella of 'immaterial labour'. It is only contextually understood as different from monetized new media work, as highlighted by the prosumer occupying both roles at once. This second category deals with the labour required for successful participation in social networks by the users of social media themselves. Writing and clicking tasks, updating one's status, commenting on other people's content, distributing pre-existing content, adding +1 s or "likes", or whatever type of interaction a particular social network calls for, make up an eclectic range of work-like activities, all carried out through the process of interfacing with technology. It is becoming clear that the idea of immateriality is both too broad and, perhaps counter-intuitively, too literal to really explain the work of the social media participant. Some aspects of immaterial labour can be applied to the labour of the social media participants, like the notion that social media work is work not commonly recognized as labour (Lazzarato 1996, n. pag.). This observation has also been made about online activities in general (Terranova 33, Paasonen 1302) and social networks specifically (Coté and Pybus 94). Who amongst Facebook users considers the time they spend liking people's statuses, commenting on photos, spreading memes, and strengthening friendships, work? These are activities which equate to or constitute types of labour through thinking, clicking and writing. Often, the notion of affect is attached to this type of immaterial labour, but this notion of the affective is added onto the immaterial, describing a perceived social result or benefit to the activity rather than distinguishing it from other types of work. This idea of affect will be returned to later. For now, it should be remembered that the notion of affect can be applied to any type of work, and is not in itself adequately unique to distinguish this second strand of online work.

There is a danger here of employing the concept of immaterial labour uncritically: writing about labour in online settings without reference to the immaterial appears to have become almost unthinkable, even in situations where immateriality does not appear immediately relevant. A clear example of this can be found in Marianne Martens' "Transmedia teens: Affect, immaterial labour, and user-generated content" (2011), where the term "immaterial labour" is used exactly twice: in the title, and within 
the reference section as part of the title of a different publication. This raises questions about the intent behind employing the term - was it simply unthinkable to leave it out? Furthermore, Mark Coté and Jennifer Pybus propose that immaterial labour as a term is insufficient: in order for it to apply fully to new media contexts, it must be updated to "immaterial labour 2.0" ("Learning to Immaterial Labour 2.0: MySpace and Social Network" 89). They define this 2.0 version of immaterial labour as "more accelerated, intensified, and indeed inscrutable variant of the kind of activity initially proposed by Lazzarato" (89) and support their argument with an analysis of the practices of MySpace users. This addition of the 2.0 raises some conundrums: if immaterial labour is not a term that adequately covers the labour in online spaces Coté and Pybus wish to explore, what does the addition of this 2.0 really help clarify? Does it merely indicate what would otherwise be clear from context - that this discussion relates to new media? How does that help define the term?

While the justification of the 2.0 add-on may fall short, Coté and Pybus are thorough in their definition, clarification and refinement of the term immaterial labour itself. They use Lazzarato's correction (in response to misinterpretations of his original article) that immaterial labour "produces first of all a "social relationship"' (qtd. in Coté and Pybus 95), and this production makes visible the social value of labour "which material manual production "hid"”' (qtd. in Coté and Pybus 95). This current research project will utilize this concept of the social value of labour extensively, while simultaneously questioning the accuracy of the notion of "immateriality" as an explanation for how these social values are formed. As possibly evident from the paragraphs above, the umbrella term of immaterial labour is fraught with inconsistencies and a general lack of clarity, as it has been extended and applied far beyond Lazzarato's initial scope. The labour of social media users seems not so much primarily immaterial but more social; immateriality is an interpretation of the way this work is carried out. Interfacing, on the other hand, describes the way this work is actually carried out, and is perhaps the more crucial issue here. Looking to Ravelry, the site makes its connection between the tangible, worldly object and the 'immaterial' social network possible through this process of interfacing.

As a final point to this discussion, it should be noted that immaterial (or perhaps social?) labour cannot easily be separated from capitalist, economic ties, as spaces 
where social labour takes place are typically also at least partially commercial spaces. This is also true on Ravelry, where social space, labour place and marketplace overlap. Lazzarato's clarification indicates that social ties are the core of immaterial labour, and capital or economic relations can only be formed on top of this existing foundation of social relations, though Lazzarato here speaks in the context of a workplace. However, most social networks encompass some degree of commercialism where social network participants make up a market available to advertisers, and the blurring of lines between producer and consumer adds a further connection between the social and the commercial. Capital ties are important and visible in social networks, as will become clear in the analysis of Ravelry, and it has been noted that these ties may even become exploitative through a reliance on social connection in order to scavenge potentially profitable user generated content. See, for example, Marianne Martens' “Transmedia teens: Affect, immaterial labor, and user-generated content" (2011) where she warns against corporate exploitation of user-generated content in the context of teens' participation in online Young Adult fiction communities, where teens discuss, respond to and even contribute their own YA fiction. To the teens who write fiction for each other, they are creating and maintaining social ties while being entertained and informed; to the publishers they are receiving free market research, valuable feedback for better targeting their young adult audiences and even free user-generated content (Martens 65).

\section{A brief discussion of affect}

Extracting the economic from the immaterial is a messy, and at times impossible, business. Surely if the role immaterial labour plays is simply to act as a foundation for capitalist exchanges, the immaterial would hold little appeal to users of social media? What is it that binds the interpersonal and the economic together in online 'work'? In scholarly literature, the concept commonly used to explain the meaningfulness of immateriality is the notion of affect. Affective labour is talked about hand in hand with immaterial labour in online contexts, often to the point of inextricability. As mentioned previously, affect is a difficult concept to pin down. Antonio Negri and Michael Hardt are commonly credited with the most widely used definitions of both affective and immaterial labour in their books Empire (2000) and Multitude: War and Democracy in the Age of Empire (2004), where they respond to and critique existing lines of thinking 
within Autonomous Marxism and feminism. They argue for the increasing importance and prevalence of both types of labour as driving forces behind contemporary (or, as they call it, postmodern) global economy (Empire 285). Their oft-cited definition of affect hinges on a vague sensation or emotional experience, and is difficult to interpret in any concrete sense:

Unlike emotions, which are mental phenomena, affects refer equally to body and mind. In fact, affects, such as joy and sadness, reveal the present state of life in the entire organism, expressing a certain state of the body along with a certain mode of thinking. Affective labor, then, is labor that produces or manipulates affects... (Multitude 108)

In other words, affective labour is labour which expresses a state of being, both physical and mental, which in turn brings about a change in mind-body state. Presumably, this change occurs in both the worker and the labour-recipient, should there be one. This definition sets affect apart from emotion, though bringing about an emotional response is certainly part of affective labour's presumed results. Hardt and Negri use the idea of "service with a smile" (Multitude 108) - manual, mundane labour carried out with the expectation that the labourer will ensure a positive emotional experience for the customer, thereby producing an 'immaterial commodity' - as a clearer example of how affective labour is carried out in an everyday situation. The worker must here make an emotional investment, even in the form of 'faking it', in order for the transaction to be complete. This is perhaps a more concrete explanation of how affect relates to labour, but it also raises some questions about how affect applies to the types of online labour discussed so far. Affect, or producing positive emotional experiences, can be located for example in Facebook's social dimensions where people reinforce friendships through the processes of commenting on, "liking" and sharing content. By interfacing with the social network, participants can uphold and strengthen notions of community by building up their own social capital, as well as reinforcing and building up the social capital of others. Perhaps this can also be thought of as types of 'immaterial commodity', where Facebook users are willing to put in a great deal of work through interfacing in order to maintain and strengthen their social realities online. 
Hardt and Negri talk specifically about affect in the context of waged, professional labour. Their work, as that by Lazzarato, and all the writings on the "new media worker" discussed previously, privilege a particular type of work quite different from, for example, the writing and clicking done by a teenager on Facebook or the offline-to-online labour of a knitter on Ravelry. This inconsistency has been noted: in their special issue of Ephemera (2007), editors Emma Dowling et al observe that “...whilst the concepts of immaterial and affective labour... succeed, to a certain extent, in describing real and existing tendencies, their analyses have taken particular forms of this labour as de facto 'advanced' forms of all the others" (2). Moving past the year 2007, the notions of affective and immaterial labour have been incorporated in a broad range of Internet-related scholarly work as demonstrated previously. The danger now, perhaps, is applying these terms too widely and too uncritically. Thinking about immateriality and affect through the lens of interfacing helps clarify and anchor these abstract concepts in observable actions of community-building.

\section{Facebook: the all-you-can-network social media buffet}

While the "immaterial" labour components of clicking, writing and sharing remain much the same within all social networks, other types of work vary depending on the network's particular focus. As there are many types of social networks, there are also many types of social network labour. The scope of social media is ever-expanding: while networks like Facebook cast a wide net in terms of attracting participants, social networks tailored to niche audiences are becoming more common. These niche social networks are typically activity-focused and interest-based. That is, people seek out tailored social networks that reflect their existing hobbies and activities in order to connect with like-minded enthusiasts, as well as the generalized networks "everyone" uses to maintain existing social ties and connections (boyd and Ellison 210). It is important to distinguish between the general and the specialized network in this exploration of labour, as this enables a more refined and accurate discussion. In order to understand what these different labour investments are and what they mean, it is useful to undertake an analysis of some representative networks and the literature associated with them. Getting a clear idea of just how varied and diverse social media can be enables a more constructive talking-through of issues of labour, and avoids a toocommon lumping together of all SNS as being more or less the same. 
So what is the distinction between a general and a specialized social network? Key to answering this question is understanding the sense of purpose participants express through their activities in different social networks. A clear example of a social network that has moved from the specialized niche to the giant mainstream is Facebook.com. Initially a closed network only for Harvard students with a harvard.edu email address (boyd and Ellison 218), over time the popularity of the network, and the demands of its user base, turned it into an open network anyone can join. It is generally accepted that people go on large sites like Facebook.com not to find new friends or social circles, but to strengthen and maintain their existing social connections both onand offline (Ellison, Steinfield and Lampe 1165). By contrast, many niche sites capitalize precisely on people's desire to find others who share their particular interest and participate in an activities-based community, often within the same geographic area.

All-encompassing networks like Facebook.com tend to only require online work such as writing and clicking tasks, accomplished directly through the use of an Internetenabled device. There is very little one can do on Facebook itself which falls outside writing or clicking - status updates, posting links and photos, playing games and chatting all fall within these two categories. As Facebook has grown, it has become a social network not requiring any particular niche interest for participation; it exists as a non-specific network in its own right. Being "on Facebook" has become an activity needing no further explanation to those who are already doing it; the act and process of being "on Facebook" is "tightly integrated into the daily media practices of its users" (Ellison, Steinfield and Lampe 1144) and holds widely understood social and cultural meaning among those already familiar with social media. The movement into an allencompassing, stand-alone social network is also reflected in Facebook's technology. The technical know-how required for posting multimedia content is now minimal, which has also reduced the labour component of these activities to only a few clicks in most instances. The idea seems to be that if you have the knowledge and literacy necessary for joining Facebook, everything else should ideally follow without a steep learning curve. This no doubt contributes to Facebook's popularity, as limited familiarity with social network technology is a smaller obstacle than in other, more specialized social networks. All one needs to become a Facebook user is an internetenabled device, an email address and ideally some friends who are already on Facebook. 
In terms of social media, these requirements are minimal. The way Facebook facilitates sharing of other digital content makes it relatively easy to contribute by posting links to external sites, re-posting other people's status updates and posting/watching videos. This is particularly valuable to those disinclined to share mini-narratives about their offline lives, though most users would presumably post a mixture of status updates and pre-existing content. A selective process underlies the sharing of existing content; "which links should I post and what does my posting them say about me" may be as important a deciding factor for sharing a web page as "which events from my everyday life should I narrativize, and what do these narratives say about me". Sharing existing content, then, is arguably as important for self-representation as updates about one's offline life. Unlike Ravelry, where physical objects must exist before they can be digitized and shared, Facebook does not necessarily require a great deal of overlap between the online and the offline in the context of reposting existing digital content.

However, it should not be downplayed that a big part of being "on Facebook" involves posting mini-narratives about one's everyday life. This necessitates a connection to the offline world, as communicating one's existence beyond the social network is important for building and maintaining social capital. This often takes place in the form of pictures, or automatically adding one's location, based on the GPS coordinates of the user's smartphone, to a status update. The process of "checking in" at a pre-set destination (appearing as a stand-alone event in a user's timeline: "so-and-so is at such-and-such location" with an added timestamp) also connects the Facebook user to physical presence and lived experience. The point here is not that Facebook is somehow more disembodied than other types of social media. Disembodiment is not a helpful term when physical presence is necessary for interaction with technology. It therefore cannot be argued that being on Facebook is less tied to materiality than other social networks. It can, however, be argued that the necessity of undertaking offline labour specifically for the purpose of participating in the social network is not part of Facebook's underlying dynamic. In other words, Facebook users presumably post about things they would be doing anyway, rather than doing things in order to post about them. 


\section{The specialist social network and kinetic self-representation}

If Facebook is primarily sedentary and not necessarily reliant on physical activity beyond writing and clicking, its counterexample can be found in geocaching communities, with geocaching.com as the predominant example. Geocaching can be thought of much like a complex and often physically challenging treasure hunt (O'Hara 1177). Participants with a GPS-enabled device travel through physical space in order to locate pre-set coordinates where they find a small item (often only a weatherproof box containing a log book) called a cache. When such a location is found, the geocacher typically logs this in the logbook while announcing the find to a wider community through an automatic notification (if hunting with a smartphone). Sometimes this is followed up with a more in-depth message or blog post including notes on, for example, how easy the cache was to find, problems encountered underway, how pleasant the hike was, and so on. Often, caches consist of nothing more than a physical marker like a plastic box, though some caches will contain a small gift (usually a low-value trinket) for the next person who comes along (Schlatter and Hurd 28). Caches can be placed and found anywhere geocaching rules allow, which only excludes places where geocachers may themselves be at risk, violate laws, pose a threat or otherwise be a nuisance. This means geocaching is well suited to the outdoors and does not need to be confined to urban landscapes; many geocachers seem motivated by the prospect of hiking through wilderness, forests and farmland - with their smartphones - as a contrast to urban living.

Of particular interest here is how academic work on geocaching has made observations about an online-offline dynamic similar to that which is present on Ravelry. 0'Hara (1178) has noted the tendency for scholarly work on geocaching to focus too closely on the bodily experience of the treasure hunt, and the way technology enables this, without taking into account how this translates into social participation online. He calls for further study into the connection between the on- and offline, and suggests that any analysis of geocaching practice is incomplete if the online community dynamic is unaccounted for. Within geocaching communities, O’Hara notes, “[On-line] participation shapes the in situ experience and vice versa" (1178). This is strongly reminiscent of offline, manual production being the entry token into Ravelry's social world, but flipped on its head: with Ravelry and similar sites, existing academic work 
tends to focus on the more ephemeral online community experience, while excluding or minimizing the significance of the Raveler's offline handicrafts practice. Work on geocaching, however, looks first and foremost at the offline physical investment into the hobby and takes this as a given necessity for participation on geocaching.com and similar. Not only does this comparison expose gaps in literature, it also highlights the very invisibility of the type of offline work Ravelry is built around.

The example of geocaching is interesting as it allows for what can be thought of as kinetic self-representation: where the geocacher goes, and the fact that the geocacher is going there, says something about who the geocacher is. This then becomes a reflexive process, in which self-representation is taken into account when choosing geocaching sites, and geocaching sites are decided upon based on what they may say about the person who visits them. This is indicated by, for example, the way caches are assigned both a terrain rating, based on factors such as ease of access and complexity of surrounding terrain, and a difficulty rating based on how tricky it is to locate the cache once the correct coordinates have been reached (geocaching.com "Glossary of Terms"). Geocachers expand upon the difficulty and terrain ratings by adding their own thoughts on geocaching.com about the process they went through while searching for and finding the cache. The process of finding a cache is typically narrativized, often highlighting the geocacher's shortcomings as well as triumphs, and these mini-narratives are typically signed off with a TFTC - Thanks For The Cache. Caches therefore mean something beyond being a specific location. The very labour of going somewhere and finding a cache, then, becomes a prerequisite for "writing oneself into being" (Sundén 88) or narrativizing one's offline, worldly experience for the purpose of self-representation, translating bodily experience and in situ reality into the language of social networks. Ravelers knit themselves into being, while geocachers hike, climb, search and rummage their way into their online community.

It can be argued that Facebook relies on similar lived experiences, as these provide fodder for status updates, photographs, discussions of participation in mutual events and so on. However, there are some major differences in terms of labour investment in these two social networks. As Facebook is general, perhaps generic, it does not require much in the way of work done specifically for the social network. Instead, Facebook users typically talk about things they would have been doing anyway, 
referencing their life as it exists outside social media. The ability Facebook users have to keep content exclusively in the online domain, such as by sharing other people's videos on YouTube, linking to other web pages and so on is also significant; such content will be a prevalent feature of any average Facebook user's daily timeline. This reposting of existing content certainly serves a purpose and contributes to the accumulation of social capital; it would be inaccurate to dismiss links and reposts as throwaway or less significant as they do not reference lived, offline experience. Facebook, therefore, is an example of how a social network can require a large degree of "immaterial" labour without resting on the same degree of physical labour as geocaching, which requires a great degree of kineticism in addition to stationary writing and clicking tasks.

\section{Locating Ravelry}

So where does Ravelry fit into this discussion? It seems that Ravelry would fall somewhere in the middle, between the generalized Facebook and the specialized geocaching. As both a social network and a resource for manual production, Ravelry's marriage of the material and immaterial through its labour space distinguishes it somewhat from other social networks. This is by no means an indication that Ravelry is unique, as shown by the comparisons above, but Ravelry seems to fill an otherwise unoccupied niche. This point of difference stems from Ravelry's immersion in handicrafts, which carries a particular historicity and social context. Again, Ravelry is not the only handicrafts-focused social network in existence. A popular alternative is craftster.org, which crowns itself the "largest online community for what is now commonly known as "indie crafts"," and explains that the word "craftster" is a mash-up of crafty and hipster, while referencing previous online communities which have also employed the -ster suffix (craftster.org "About"). Craftster.org does not limit itself to certain kinds of handicrafts like Ravelry, and is much more generalized in its content. The key quality of an item on Craftster lies not in its type or in its underlying labour, but in its "indie-ness". Craftster is therefore quite different from Ravelry as it presupposes a specific identity; simply signing up to the site identifies one as a "crafty hipster". This seems discursively different from Ravelry where the expected identity is less overtly socially prescribed; potential members are simply referred to on Ravelry's front page as "knitters and crocheters". ${ }^{8}$ 
The absence of an easily pinned-down presupposed identity already says something about the role of labour on Ravelry. Self-representation is not guaranteed merely from signing up; one does not become aligned with a social movement or subculture just by having a presence on the site. What one does once on the site therefore becomes all the more important; one's labour space must be filled up. Much of the offline labour Ravelers put in contributes towards their cumulative selfrepresentation, which in turn becomes the foundation for social capital. Understanding where and how self-representation happens is therefore a necessary cornerstone in this analysis of social media labour.

\section{The prolific profile?}

In their definition of social network sites, boyd and Ellison (2008) establish as their very first point that the ability to create a "public or semi-public profile within a bounded system" (211) is intrinsic to a social network. This is certainly true of the sites they predominantly reference in their article (Friendster, Facebook and MySpace), and is accepted as a mainstay of social network dynamics. The profile page is where participants provide a snapshot of themselves to both existing and potential friends, and is therefore inherently self-representative. boyd and Ellison's definition of social network sites rests heavily on the profile page as the distinguishing feature identifying a SNS, and they establish SixDegrees.com (launched in 1997) as the earliest recognizable SNS based on its inclusion of personal profiles (214). After establishing the need for a profile page, boyd and Ellison include within their definition that a social network site must "(2) articulate a list of other users with whom [the original user] share[s] a connection, and (3) view and traverse their list of connections and those made by others within the system" (211). In other words, a participant must have a profile page, this profile page must list connections the user has to others in the network, and each connection must lead to a different profile page where more connections can be seen.

But having the definition of a social network site rest on the importance of the profile page can be problematic. As mentioned in chapter 1, Ravelry.com provides the option for users to fill out a page called their profile, but this very rudimentary single page is not the nexus of social connections on the site as it does not highlight or showcase a user's contributions to Ravelry. It is not uncommon to leave one's profile 
page as blank as possible, including only default information which cannot be edited out. Currently, the only mandatory fields that are always completed and displayed in a profile are the username, the date the user joined Ravelry and a brief snapshot of the content of the user's Notebook. Other fields in the profile, such as date of birth, location and likes/dislikes, are optional. Ravelry even draws attention to the profile's voluntary, dismissible nature by describing these "personal bits" as "just for fun - all optional..." on the page users access to edit their profile, while using no such language anywhere in "My Notebook", in which users' projects are stored. This casual approach to the profile tells us that the profile itself is here not viewed as an essential component of social network participation; this is not where investments in self-representation are made. What the Raveler is is not as important as what the Raveler makes. Rather than being the focal point of social connections and displays of labour, the profile points to the social space and labour space found in "My Notebook". The labour component of completing one's profile is minimal: one can simply accept Ravelry's defaults without further editing after the initial registration, and then disregard the profile entirely without noticeably impairing one's ability to participate in the wider community. Default values, such as number of friends and contents of one's Notebook, update automatically. Most importantly, when navigating parts of the site where one may encounter other users, following the hyperlink in their username will more often than not lead to that user's Notebook, not profile. This is written into Ravelry's source code by the web developers; Ravelers do not get to choose where Ravelry links to their profile and where it links to their Notebook. In this sense, the Notebook has taken over the role of the profile despite the profile existing as a separate entity. Both the profile and the Notebook fulfil boyd and Ellison's criteria through their articulation of the Raveler's connection to other Ravelers (211). Yet, the traversable list of friends and other connections is not readily drawn attention to in "My Notebook", which displays 'projects' first by default when navigating to a user's Notebook by clicking on their username. This suggest there is more at work here than a simple renaming; "My Notebook" is not simply "My Profile" by a different name, and "My Profile" showcases "My Notebook" by drawing attention to where records of a Raveler's labour can be found. 
The evident disinterest in making the user profile important to the Ravelry experience sometimes leads to it being overlooked altogether. Jill Rettberg, in her article "Freshly Generated for You, and Barack Obama': How Social Media Represent Your Life" (2009) appears to have missed the actual profile page entirely, identifying instead Ravelry's "My Notebook" as the user profile. Here, "My Notebook" has replaced the profile as evidence of self so fully that the profile becomes redundant, throwaway or in Rettberg's case, entirely unnoticeable. This misidentification is valuable as it illustrates where Ravelry breaks with the "typical" flow of a SNS. This is also where the discussion of profile pages intersects with the concept of labour. Much of the labour undertaken on Ravelry - in part, through the more material labour of knitting and crocheting - and in social media in general is the work of self-representation.

Ravelry is a particularly clear example of the connection between social network and labour. Its connection to offline space makes it distinguishable from other social networks, while maintaining a fairly standard social network dynamic in its online realm. By being both like and unlike other social networks, it allows for conclusions to be drawn about dynamics found across the sphere of social media, while making these dynamics explicit and visible within its own special flow of offline-to-online work. Labour matters on Ravelry, and Ravelry is much like many other social networks. Therefore, Ravelry can be used as an example for illuminating the importance of labour as a function of SNS in general. As discussed, there are many parallels between the writing and clicking tasks necessary in big, all-encompassing networks like Facebook and specialized ones like Ravelry. Ravelry then goes a step further by anchoring these tasks of interfacing in physical, material labour, and devoting an entire sphere of interaction to this labour via the site's labour space. This project takes a step towards illuminating the role labour plays in social realities online, using a clear but relatively niche example.

In the area of online handicrafts communities, there is no wealth of academic material to draw upon. The most detailed studies of Ravelry have been undertaken by Australian scholar Sal Humphreys in the form of conference papers and journal articles, with particular reference to the economies of social media ("Ravelry on the Net: a social network market?" 2008, "The economies within an online social network market: A case study of Ravelry" 2009). Humphreys covers many of the areas already discussed in 
these two chapters, but her analysis is centred on Ravelry as a type of marketplace, which is only one of three spaces examined in this project. This is valuable as Humphreys picks up a trail which cannot easily be covered within the scope of this thesis, but is nonetheless vital to a full understanding of the complexities of Ravelry. Her work will be drawn on particularly for chapter 4, where the relationship between handicrafts commodity and social network will be analysed. Humphreys is also one of only a few scholars who have published about online handicrafts communities in general, with her 2008 Continuum article "Grassroots creativity in new media environments. Yarn Harlot and the 4000 knitting Olympians". However, moving straight to the relationship between Ravelry as marketplace and the accumulation of social capital overlooks the role affect plays, both on the site itself and within the wider context of visible domesticity on the internet. As highlighted in this chapter, immateriality is often employed somewhat uncritically when discussing labour online. Knowing that affect and immateriality are often used concomitantly, and that what is described as immaterial is often deeply rooted in the physical, kinetic and embodied contradicting the very definition of immateriality, where does this leave affect? Is it similarly contradictory? While one aspect of Ravelry's labour relates to the presumed immateriality of work that takes place online, another aspect is the more tangible domestic crafts of knitting and crochet. These activities, and the associations with domesticity, 'women's work' and caring which surround them, significantly predate any online community. Yet, this type of labour has also been absorbed and made meaningful within an 'immaterial' setting, highlighting the need to understand how this more 'affective' labour has become socially meaningful on Ravelry. 


\section{Chapter 3 - Hip domesticity and the cloak of affect}

\section{Introduction}

While affective labour can theoretically be encountered anywhere, it is more typically associated with certain types of production, and is strongly associated with domestic labour. Affect is understood as something which expresses and produces an emotion, and affective labour is labour that produces a kind of emotional exchange, often in a rewarding and positive fashion (Multitude 108). Putting one's domestic work on display can be read as a process which reflects affective relations where emotional outcomes have been achieved through acts of domestic labour. While domesticity was once hidden, considered uninteresting and trivial to the public eye, it has become increasingly visible to the point where home-makers and other practitioners of domestic arts are now actively writing about and recording their everyday domestic work through a range of media. Conventionally published best-selling books, with titles such as The Secret History of Domesticity (McKeon 2005), Radical Homemakers (Hayes 2010), The Gentle Art of Domesticity (Brocket 2008) as well as Debbie Stoller's pivotal Stitch'n'Bitch (2003) make it clear that domesticity and domestic arts have become relevant in the public eye. It is also clear that this new domesticity translates well into online mediascapes: blogs, social networks and other online arenas form popular and accessible platforms for community-building based on domesticity and handicrafts.

The purpose of this resurgence of "visible" or publically discussed domesticity, which is occurring both on- and offline, appears to be associated with a progressive gender politics where 'women's work', once considered trivial, is made important and publically accessible. Here, making domesticity a topic worthy of public engagement is associated with ideas of liberation and social change. This rekindled interest in domestic work and visible domesticity as a platform for social change is often referred to as "new domesticity" (Railla Get Crafty 2004). Ravelry and its peer sites have come into being during this new domesticity period, which some see as a more or less deliberate present-day revival of the domestic arts, aimed towards reigniting public interest in all things homemade, thrifty and crafty for the purpose of forming a "social movement" (Railla "New Domesticity" n. pag.). 
However, thinking of new domesticity in terms of these 'resistance themes' (Minahan and Cox 11) where acts of domesticity are at some level politically motivated does not fully account for the wide range of sites and communities where "new" domesticity is present, such as the LiveJournal.com community "Hip Domestics" or online housekeeping community FlyLady.net. This display of domestic labour is also at Ravelry's very core, but clearly expressed socio-political motivations are harder to come by. What Ravelry and the sites mentioned above do demonstrate, however, is a relationship between domesticity and fashion. This does not negate the presence of affective meaning or the potential for social change; rather it broadens the scope of "new" domesticity and incorporates the domesticity of those who are not participating in new domesticity as social movement. In order to account for this complexity, it is helpful to think of not only what is new in new domesticity, but also what is hip.

\section{A modern history of domesticity}

The development where previously unrecognized or undervalued types of labour are slowly being uncovered is also related to affect. This is particularly true of domestic labour which typically takes place in the private sphere, such as the knitting on Ravelry. Jack Bratich $(2007,2010)$ draws lines between craft culture, or "fabriculture" as he calls it, social movements, activism and cyberfeminism. Sal Humpries analyses the specifics of amateurism, creativity and social network economies within the context of online knitting communities (2007, 2008, 2009). Minahan and Cox Uournal of Material Culture 2007) assert that the presence of modern-day informal knitting groups and communities, combined under the collective term "StitchnBitch" (after Debbie Stoller's 2003 book by the same name), "can be seen, in part, as a negative response to major political, social and technological changes including globalization, terrorism, damage to the environment and the dislocation of the Information Society" (5). While these scholars approach fibrecrafts and technocultures from very different perspectives, what they have in common is that their work to varying degrees interacts with the concept of "new domesticity", albeit without necessarily using the specific term. New domesticity (as opposed, somehow, to "plain old domesticity") is thought of as a resurgence in interest in old crafts and domestic arts, particularly those which have historically been the domain of women. There is no definitive list of activities or hobbies thought of as particularly "newly domestic", but fibrecrafts, baking, canning, thrift and reduction of 
the consumption of store-bought commodities are recurring themes throughout the new domestics blogosphere. The new domesticity trend is also intrinsically linked to modern technology; the "Information Society" Minahan and Cox refer to above is the very same society these communities depend upon. This online presence is perhaps new domesticity's most easily identifiable attribute: having an interest in "old" domestic arts is only half the picture, the other half is the documenting, asserting and displaying this interest in online communities. This blurs the boundaries between domesticity as something private, set outside the public sphere, and the making-visible of private lives common in social media through practices such as blogging or posting mini-narratives in the form of status updates within a SNS. New domesticity is open and visible, it is there to be engaged with, commented upon and criticized. As Bratich and Brush observe in "Craftivity Narratives", this "resurgence of the "private" into the public sphere... reopens the politics of domesticity" (1). Clearly, new domesticity did not arise out of nowhere. So what is the context of its emergence?

Until recent history, that which took place in the private sphere under the umbrella of domestic labour - historically viewed as the work of women - was considered a matter both too mundane and too private to hold a place of any significance in public debate and discourse, which belonged within the sphere of men and masculinity (Cowan 19). Household labour was relegated to an inferior status, a necessary form of labour but not one comparable in value to monetized employment (Cowan 210). It is no coincidence that household labour fell almost exclusively within the domain of women. The term "women's work" seems to have been employed to simultaneously categorize and dismiss domestic labour as not belonging to the highervalued class of work undertaken outside the home, while still expressing the significance this work played, and continues to play, in women's lives (Cowan 8). The chores and tasks that primarily take place within the domestic sphere for the purpose of maintaining and improving the home, while often also running and raising a family, have historically occupied much less space in public discussion and record than the tasks and responsibilities of men. This topic of the invisibility of women's labour began to gain academic clout in the latter half of the twentieth century, when greater attention began to be paid to the social, personal and economic consequences of the invisibility of women's work (Cowan 8). The very fact that so much of the lives of half the world's 
population had gone largely unstudied, undocumented and unnoticed became a starting point for extensive academic research. Histories of domestic, everyday labour, such as Ruth Cowan's More Work for Mother (1983) and Michel de Certeau's pivotal The Practice of Everyday Life vols 1 and 2 (1984/1998), are a starting point for demonstrating this growing interest in studying domestic daily life in the latter third of the $20^{\text {th }}$ century.

As scholarly work on the making-visible of women's histories and domesticity accumulated, so did domesticity-focused literature and media gain popularity among the general public. Early celebrity chefs like Julia Child became mainstays of television blazing the trail for TV personalities like Martha Stewart, who gained significance throughout the West as a symbol of all things homemade and domestic, while New Zealand's own celebrity chefs Hudson and Halls ran on television from 1976 to 1986 before moving to the UK (nzonscreen.com). These sources of 'broadcast domesticity' were and are public in nature, setting them to some degree apart from earlier sources of domestic advice which were typically printed in the form of manuals or magazines aimed specifically at women, and contained within the home (Cowan 177). Through the process of incorporation into broadcast media, domestic crafts once considered private, isolated, lowly and "perceived [as] minimal contributions to cultural development" (Minahan and Cox 11) became assimilated by popular culture, drawn on for entertainment and for the selling and consumption of commodities.

Chefs and cooking have perhaps received most attention in popular culture, and as such they provide an easily recognizable point of entry into domesticity as conspicuous activity. Indeed, The Practice of Everyday Life volume 2: Living and Cooking (1998) provides an insight into domesticity centred on meals and cooking through transcripts of interviews and conversations with French homemakers (most of them women) who paint a detailed picture of the finer nuances of their domesticity. Throughout the book, the women interviewed allude to the "affective investment" (151) they make through their domestic labour when describing what they do in order to make their homes spaces where people gather in order to enjoy a particular warmth and atmosphere. These descriptions belong within de Certeau's larger project of accounting for everyday behaviour as something other than strict patterns of production and consumption: the trip to the grocery store is not simply an account of 
consumptive behaviour, but a narrative of small decisions so mundane they are mentally automated, but which still have consequences in the private, everyday life and constitute public displays of consumption $(74,81)$. Ravelry, and the recording and displaying of finished objects, seems to run parallel to this process of documenting a slice of domestic daily life. Ravelers are given the opportunity to talk in detail about the minutiae of their knitting, much like Luce Giard gave French women the opportunity to talk in detail about their cooking (159). The templates Ravelers must fill out in order to complete records of their finished objects ask for specificity while leaving room for the individual Raveler to narrativize their projects. This combination of specificity and narrative is central in new domesticity.

\section{Affective labour explained}

Before moving on to the analysis of the emergence of domesticity in new media as well as Ravelry's affective dimensions, affective labour must be put in context and evaluated because it is a recurring phenomenon within hip domesticity. As touched on previously, affect is typically employed to explain a meaningfulness of labour which goes beyond the material, economic or practical. The affective is understood as that which expresses and produces an emotion, causing a change in the mind-body state (Multitude 108). The affective aspect of labour is the interpersonal exchange which takes place human to human, even if these interactions are mediated through interfacing. This social meaning appears to be carried across practically all types of labour: Hardt and Negri's idea of "service with a smile" (Multitude 108) goes beyond describing affect as a mere adornment of an otherwise impersonal exchange of labour

for goods and services, and leaves space for this not to merely be an add-on, but to hold social meaning. Rather than presenting affect as the thin veneer of positive emotion concealing the discontentment of the hospitality worker, Hardt and Negri allow for affective dimensions in both the worker and the recipient of this labour. The friendly smile, this brief presentation of a mind-state generally interpreted as warm and approachable, could serve not simply as fulfilment of duty or response to expectation, but as a way for the worker to inject a small interpersonal exchange into their labour which it might have cost energy and effort to suppress. Affective labour, therefore, is labour which brings about an emotional effect - usually a positive one. Within social media, affect can also be thought of as that which is present within labour which 
renders it socially meaningful. On Ravelry, affective meaning is found in the creation of digitized mirrors of manual handicrafts labour. These records of labour carry meaning in their digitized form as evidence of care-work and as interaction with the wider online community, but they also speak to the potential of warm, fuzzy knitted objects to act as symbols which bring about positive emotional changes.

As most of us are social beings, it could be that it is simply quite difficult to perform any kind of labour without interspersing this work with social qualities. The same is perhaps true of the way affective labour is employed as a term within new media. Affect is attached to the immaterial as a way of making sense of why interfacing seems so important, when this interfacing seems to hold little practical application. Why do Facebook users carry out repetitive clicking and writing tasks, interfacing with the social network in order to connect and share with people they are likely to have face-toface conversations with anyway? Why does the knitter post pictures of the sock she ${ }^{9}$ has made, complete with details about what materials were used, what date it was completed, and who the sock is for? And why does the geocacher need to announce electronically that she has found a cache? None of these things appear to serve any offline, practical purpose. These events would perhaps have unfolded in exactly the same way without interfacing. The sock would probably still have been painstakingly hand-knitted and gifted, and friendships would most likely have survived one party not having clicked "like" on a Facebook update. The knitter would have gained exactly the same bodily, manual experience from completing the sock if she had not interfaced to tell the social network about it afterwards, and the process of knitting the sock may still have been a pleasurable, positive experience. This is where the significance of the work we do both off- and online becomes clear: very little of the Raveler's labour online makes sense without considering its social meaning and its place within Ravelry's social space. Assuming that a sock is not fully a sock until it has been validated by a wider community seems extremely peculiar. Ravelers have found a way of translating manual labour offline into an affective reality within their online social space, and through this translation they reap positive benefits in the form of community-building and social capital. Affective meaning therefore depends on context: labour which is socially meaningful in one community may be meaningless in another. So can something defined as inherently subjective really be observable? It is not as straight-forward as stating that 
handicrafts labour is affective labour, or social labour is affective labour. Affect is ephemeral, so closely tied to and dependent upon a particular context and a particular subjectivity that it cannot easily be pinned to any one type of labour. It can, however, be understood as something which makes labour socially meaningful.

\section{Domesticity vs. commodity?}

Matters which were once private and mundane are now fodder for both popular mass media and smaller online communities - so how is this visible domesticity different from new domesticity? Is there any true difference? While new domestics who take a hard line against capitalism may rally against the type of commercial and simplified homemaking present in broadcast media and advertising (see Shannon Hayes' Radical Homemakers: Reclaiming Domesticity from a Consumer Culture 2010), commercialism is undeniably present and at times even central within new domesticity. The tension between domestic life and commoditisation has been a source of much theory and critique. Minahan and Cox (2007), for example, suggest that fibrecrafts communities provide a form of resistance against mass production, gender norms and commodification (10-11), but as will be demonstrated this view is complicated by the very observable presence of commodity culture within new domesticity generally, and fibrecrafts on Ravelry specifically.

In the blogosphere, the website FlyLady.net has become a paragon of successful, if perhaps somewhat excessive, domesticity. References to FlyLady can be found in new domesticity blogs such as Happy Domesticity and Rediscovering Domesticity as well as LiveJournal community "Hip Domestics" to name a few, and does not stand outside new domesticity as an offshoot or contrast. FlyLady speaks directly to its audience as 'ladies', indicating it has no interest in gender-neutrality. The site promises to teach women who feel "overwhelmed, overextended and overdrawn" to "gain control over their housework" so that they no longer feel ashamed of their domiciles by systematizing their domestic labour through a rigorous, time-consuming and extremely thorough programs where all household tasks are recorded, charted and tracked. Branded implements for specialized cleaning are sold through the FlyLady webstore, which is central to the site. In terms of screen real-estate, the shop appears as the first link on the site's most prominent navigation pane. FlyLady takes domesticity to extremes - no sink 
remains unpolished, no corner of one's abode may ever be a source of embarrassment. FlyLady appears to represent a regressive politics in which cleanliness, neatness and investment of time and effort into the home is equated with success and happiness, and disinterest in domesticity is associated with shame, sloth and failure to care for one's family. This starkly contrasts with Ruth Schwartz Cowan's warning in her conclusion of More Work for Mother (1983): “

\footnotetext{
We can best solve the problems that beset many working wives and their families by not returning to the way things used to be (since that is probably impossible and, in view of the way things really used to be, hardly attractive), not by destroying the technological systems that have provided many benefits (and that much of the rest of the world is trying, for fairly good reasons, to emulate), and not by calling for the death of the family as a social institution (a call the vast majority of people are unlikely to heed) - but by helping the next generation (and ourselves) to neutralize both the sexual connotation of washing machines and vacuum cleaners and the senseless tyranny of spotless shirts and immaculate floors. (216)
}

If "new" domesticity - which supposedly incorporates scepticism of commodity culture and resisting traditional gender roles - includes sites like FlyLady.net, what does this say about the meaning of new domesticity? FlyLady takes household labour to extremes and expects conspicuous consumption of domestic products, yet it is frequently referenced and utilized within a new domesticity which describes resistance against such imposing and prescriptive capitalist tradition.

As a counter-point to FlyLady's near-militaristic approach to housework as a necessity in order to express love and caring (even if one does not necessarily feel it), what emerges from de Certeau's Living and Cooking is the sense that, while timeconsuming and repetitive, domestic tasks still hold emotional and social significance in the minds of the women describing them. Consumption of commodities is secondary to this more emotive element. Luce Giard relates the sense of relief and joy these women experience when discussing their domestic routines and habits down to the smallest detail, as recounting this labour is "recounting one's self" (179). This emotional consequence of sharing the mundane and domestic seems pertinent as it appears so similar to the expressions of domesticity now found in a much wider array of media. On Ravelry, the sharing of minute details of a knitting project through "My Notebook" could perhaps play a similar role as Giard's interviews: they let Ravelers express and share details of labour which would otherwise have gone unnoticed. Due at least in part to 
this on-going effort to shed light on the significance of domestic work, we now live in a world where domesticity is far from invisible. In fact, it would not be difficult to argue that Western society is moving in the opposite direction: domesticity, or at least the ideal of domesticity, has become conspicuous. At any given time, television commercials will advertise the latest in DIY technologies, ready meals, cleaning products, furniture and so on. Gaps in television scheduling are filled with brief, advertorial-style cooking shows like Food in a Minute. Entire ranges of magazines are devoted to interior design, home décor, food and recipes, childrearing, gardening and handicrafts. FlyLady.net promises control over one's household through systematizing and cataloguing domestic work. Ravelry offers community and resources for fibrecrafts enthusiasts. Both fall under the umbrella of a trendy domesticity visible across popular culture, predominantly expressed within, but not exclusive to, online communities.

\section{Hip home ec: from cyberfeminist resistance to hip domesticity}

Attempting to identify exactly what this notion of new encompasses in new domesticity highlights exactly where this term falls short. In her blog New Domesticity, Emily Matchar cites a lack of trust in corporations and government "to keep one's family safe and healthy" as one of new domesticity's driving forces ("Where every mother is her own EPA" 15 March 2012). She expresses the belief that one's family health is under threat from the outside and that good health can be achieved through a conscious change in domestic practice. This is to some extend consistent with the resistance themes Minahan and Cox outline in "Stitch'nBitch: Cyberfeminism, a Third Place and the New Materiality" (Journal of Material Culture 2007). New domesticity as explained by Matchar seems to be about questioning and resisting biopower and changing one's habits of domestic labour because failing to do so could have harmful consequences for one's bodily self and one's family. This presumes that "standard" domesticity allows corporations and governing powers access to one's home and private life, presumably mostly through commodity consumption, and that this access is something one must become aware of and consciously resist through new domestic practice. Bratich and Brush (2007) observe something similar about knitting communities: that they are to an extent rooted in activism and cyberfeminist ideals, and arose at a time in the mid-90s where global attention was focused on unethical sweatshop practices of multinationals ("Craftivity Narratives" 4). At the same time, fibrecraft communities continued from a 
historical context where knitting groups were ridiculed as the pastimes of idle women, "gossiping circles" detached from all significance and agency (Bratich and Brush 9). Their powers combined seem to form an ideal foundation for reclaiming and resisting what Bratich calls "craftivism". But is it really that simple? Can new domesticity be read as a form of resistance and cyberfeminist activism, and left at that?

Sites like FlyLady complicate any cyberfeminist ideals underlying new domesticity. FlyLady is not interested in resisting any commercial forces governing one's domestic life, nor making any kind of activist statement about gender, labour and social injustice. On the contrary, FlyLady adopts a philosophy in which women are expected to demonstrate their worth, self-esteem and ability through maintaining a spotless home - hardly feminist fare. Within the logic of FlyLady, this is thought to give them social capital and self-esteem: keeping one's home in a state of "CHAOS" ("Can't Have Anyone Over Syndrome") is explained as a real, external problem leading to social isolation, hopelessness and becoming overwhelmed. This problem can occur in the life of any SHE (Sidetracked Home Executive, a vague term FlyLady employs to identify women who supposedly could benefit from the site's programs) and can only be overcome through near constant domestic work. There is something punitive about the rigorousness of FlyLady's cleaning regimes and self-discipline, even sleep-wake hours and dress codes are suggested by the FlyLady program - yet this extreme scrutiny is described in terms of love. According to FlyLady, "[That] shiny sink is a reflection of the love that you have for yourself" and "Nothing says I LOVE YOU like a Clean [sic] toilet to throw up in when you are sick!". The very real and intensive labour investment necessary for staying on the FlyLady program is concealed under a cloud of affective associations.

It may sound like FlyLady is an outshoot or statistical outlier standing mostly outside the world of online new domesticity, as it seems so distant from the semi-ironic StitchnBitch groups, indie craft communities like Craftster and self-identifying feminist bloggers like Emily Matchar. This, however, is not the case. One of the oldest and most active new domesticity groups on the Anglophone internet, LiveJournal's "Hip Domestics" community, ${ }^{10}$ contains a multitude of posts casually referencing FlyLady, as though it is communally accepted and recommended as a popular resource. For example, a post made in 2008 where a hip-domestics member asks for advice because 
the FlyLady program isn't working for her received a total of 70 comments, none of which ask for any clarification or explanation of what FlyLady actually is, information which is not included in the original post. ${ }^{11}$ More pointedly, none of the comments express any significant critique of the FlyLady program as one might expect if accepting the position that online new domesticity is a form of resistance rooted in cyberfeminism. So what exactly are these communities about? The LiveJournal community "hip-domestics" inadvertently hits the nail on the head with its choice of name. Leaving aside any conscious acts of resistance, irony or nostalgia, participating in online communities centred on domestic work and handicrafts has simply become trendy. Participating in communities like hip-domestics, or posting one's finished objects on Ravelry, are activities which hold social significance as an expression of preferences or tastes. Participating in these communities is to some extent a form of taste performance (Liu 2007). By contributing to Ravelry and recording her labour, the Raveler aligns herself with a specific social identity: the Raveler is participating in a community which exists within the "now", the handicrafts themselves may be ancient and the materials basic but the social identity is a current phenomenon with its own unique characteristics.

Perhaps, rather than "new domesticity", it is more accurate, then, to refer to this current revival of handicrafts and home economics as hip domesticity. The term hip domesticity is drawn from both the LiveJournal community "Hip Domestics" and the title of Jean Railla's book Get crafty: hip home ec (2004). ${ }^{12}$ Moving from the new to the hip escapes the problem of needing to define precisely what is new in new domesticity. Interpreting Ravelry and its peer sites as a reclaiming or reinterpretation of the gossipy, all-women knitting circles of the past (Bratich 9) seems too politically motivated and is not necessarily reflected in the textual object. Reading hip domesticity as part of a "modern, hip, sassy, post-feminist approach to life in a wired world" presumes the appeal lies in a "women-with-attitude" cyberfeminist discourse (Minahan and Cox 9). However, the realities of "new" domestic communities online such as FlyLady problematize this ideological definition. Hip domesticity, on the other hand, bridges the gap between domesticity, commodity and affect by acknowledging its current relevance (and potential future irrelevance) while alluding to its ties to consumer culture. This means it is not necessary to buy into new domesticity's veneer of anti-consumerism and 
anti-capitalism. Hip domesticity also provides many more opportunities for analysing online domestic communities: rather than assessing underlying activist or ideological motivations in attempting to locate the "new" within the sites, communities like Ravelry can be read as belonging within a trend, a moment in time, and this very trendiness contributes to the social meaning of the site. This opens up possibilities for analysing Ravelry as a site of textual performance - what Jenny Sundén calls "writing oneself into being" (2002).

\section{Gender: the elephant in Ravelry's living room}

Sundén's work on analysing online communities as sites of textual performance is centred on gender and gendered performances. The significance of gender in Ravelry's content has so far only been alluded to. It has been established that affective labour fits within a historicity where it is strongly associated with 'women's work', made visible through a lengthy process of exposure and exploration. Listening to women's stories and their descriptions of everyday labour, as in the work of Certeau at al (1998) continues to be central to this process. The work of the Raveler - the selection of patterns and materials, the producing of the object with its progress, mistakes, corrections and modifications carefully documented, the photographing, categorizing and publishing details of the object and the labour process, and the consequent gifting, selling or displaying the object - is documented, scrutinized and most of all made meaningful within this much wider context of domestic exposure, where the tasks which fall into the category of the offline and manual have previously been paid little attention. Ravelers here narrate their own little story of the work they have done; the architecture, purpose and social dynamics of the site's multiple spaces all work together to make the telling of these stories seem a natural, commonplace and rewarding thing. There is no need for the Raveler to stop and ask why this is all necessary. The Raveler is never asked, in any of the templates or forms she fills out to share details of her current knitting project, why she chose this project, or more to the point, why she is sharing this at all. Of course, this should not come as a surprise as Ravelry would no more ask its users "why are you here" than Facebook or Twitter would. This would be the moment where the social network is "made strange" (Bigge 2006, n. pag.): in order to interrogate it successfully, one must ask the questions its participants deem obvious or selfexplanatory. 
To this point the pronoun 'she' has been employed when providing examples of a typical or generic Raveler. This is not coincidence, nor is it to make a point about diversity and inclusion. Knitting and crochet, with is anchoring in history as women's crafts, still attract primarily women and most handicrafts blogs and communities are comprised of mainly women (Bratich 2010, Humphreys 2008, Bratich and Brush 2007, Minahan and Cox 2007). While Ravelry gathers no statistics about the gender identities of its userbase, it takes only a little casual browsing before a firm impression emerges of the site's content as primarily by, and for, women. This impression comes about through many textual channels: the first and most obvious is the widespread use of women's faces in pictorial content throughout the site. Women's faces appear in profile pictures next to usernames, in banner ads, promoted patterns, portraits of designers, finished objects modelled by their creator, next to announcements, in the Ravelry staff's front page blog and a myriad of other locations. As an example, at the time of writing (26 March 2012) Ravelry's front page, accessed immediately after logging in, displays a total of 112 individual pictures. Of these, fifty six are pictures where women's faces are the focal point in the frame. 9 photos depict women's torsos modelling knitted garments where the face is not visible, and 18 pictures are of pets and animals. The remaining shots are predominantly finished objects and profile photos containing mostly text. Compared to the 56 photos of women's faces, there are only 3 photos recognizably of men, 2 of them facial shots that mirror the style of the photos of women's faces filling up the frame, and one picture of the bearded, lower half of a man's face taking up a small space in the top-left corner of a much larger frame depicting the Ravelry mascot (a dog). Such visual analyses conducted in other areas of the site such as patterns and projects searches return overwhelmingly similar results.

It is of course neither strange nor surprising that Ravelry's members are mostly women. What is interesting is that despite the history of knitting as women's labour, and the political motivations sometimes associated with the handicrafts revival, Ravelry's gender divide is hardly ever commented upon or discussed. That the site is made up mostly of women, and that this practically all-women environment contributes to how the Raveler's labour is made socially meaningful, does not appear to be a subject of much thought or debate. In fact, the Ravelry officials seem to take great care to use gender-neutral language in their posts and announcements; no assumptions are 
seemingly being made about their userbase. Compared with other handicrafts-based networks, Ravelry relies much less on stereotypically gendered visual clues such as FlyLady's pink and purple colour scheme and identifying a single-gendered audience through consistently addresses its members as "ladies". Raverly's yellow, maroon and green colour scheme and matter-of-fact, functional fonts and layouts do not seem to make the same assumptions about its userbase's taste preferences as FlyLady's pink and purple. Ravelry is more similar to etsy.com's plain, grey and white functional webstore layout. Yet, the overwhelming presence of women's faces, names and bodies clearly identifies Ravelry as a gendered space. Gender seems to play the part of the elephant in the room, blatant but never mentioned, imposing but assumed benign.

\section{I am what I am, not what I knit. Replicating one's offline social reality through online taste performance}

As the prevalence of women's faces in pictorial content suggest, when Ravelers write themselves into being they are also writing gender. While Ravelry does not adhere to stereotypically gendered codes in its layout and visuals, the prevalence of usergenerated visual content which is clearly coded with gendered meaning demonstrates that gender forms part of Ravelry's social world. Where FlyLady is kitsch in its employment of gendered codes such as the pink colour scheme or addressing its userbase as "ladies", Ravelry attempts to remain neutral, leaving it to its members to write their own gender and create their own visual codes. Is the filling up of Ravelry's "ungendered" spaces with gendered user-generated content a statement from the site's users? As suggested by Hugo Liu and alluded to by Jenny Sundén (“Cyberbodies" 91), areas of social network sites which provide means for expressions of interests, likes and dislikes "function as an expressive arena for taste performance" (Liu 252). The textual nature of online communities leaves "even greater room for identity experimentation" as "one does not fully exist until one writes oneself into being" (Liu quoting Sundén 253). On Ravelry, this writing takes place in part through actual writing, but also through recording of materiality via digitized 'mirrors' of material objects. The creation of these mirrors can be read as performative on multiple levels: the amount of work required for knitting a finished object, then digitizing, uploading and cataloguing it is not only a process of working, it is a process of performing labour. Why is this 
performance of labour so valued amongst Ravelers? Is the fact that this is a documenting of "women's work" a contributing factor?

In Liu's study of taste performance amongst MySpace users, he notes that socioeconomic and aesthetic factors both contribute to taste, but there is good reason to avoid approaching taste from too strictly a socioeconomic perspective as this tends to lead to determinism (255). Instead, Liu acknowledges that socioeconomic and aesthetic approaches to taste tend to compete with each other, and he conducts an analysis which leaves some room for socioeconomic factors while focusing on aesthetics. On Ravelry it would be very difficult to draw any conclusions about socioeconomic differences or similarities between members based on their performance of taste, but it is possible to say something about aesthetics. What Ravelers have in common is that they carry out material, offline labour, and this labour carries affective meaning both as a hobby and an expression of a historicity in which handicrafts labour is associated with care-work carried out by women in the private sphere. Knitted objects take a long time to complete, and this need for a real-world investment of time and labour suggests that “identity experimentation" (Liu 253) online carries over to, but is perhaps also limited by, the offline . If identity experimentation is taking place here, it is taking place both onand offline. What a Raveler knits may express tastes and preferences for particular types of garments, yarns, patterns and other commodities, but the fact that the Raveler is knitting, and proving this to the social network, is in itself also a particular taste performance. What is being knitted matters, but the fact that knitting is taking place takes precedence, and this act of knitting says something about the identity of the Raveler. Where MySpace users perform their tastes through mentioning favourite bands, movies, foods etc. on their profile page, Ravelers perform their tastes through their Notebook, particularly in their 'projects' and 'stash'.

One of the functions of taste performance is to demonstrate belonging to a group or community through "group identification" (Liu 258). Multiple studies have also noted that users of social media tend to extend their offline social realities into the online; transparency between on- and offline self is common (Lee 714, boyd and Ellison 221), and those who possess social capital in offline communities tend to carry over this social capital into online communities as well by bringing their social connections with them, creating "a continuum between the physical and the virtual world" (Lee 711, 
715). Transparency is a key concept for understanding how gender is written on Ravelry. Ravelers are already tied to the material through their knitted objects, why should they not write their own selves into being with a similar degree of transparency? In other words, there may not be any political, cyberfeminist motivation behind the way Ravelers gender their user-generated content and their own textual performance despite the site's efforts to remain gender-neutral. Ravelers may simply be bridging a gap between their off- and online selves, performing themselves as gendered beings because this is the performance they already carry out in the everyday.

Thinking of gender strictly as performative, and labour strictly as taste performance, becomes problematic when viewed in light of affect. The link between gender and affect is treacherous territory. So far, the connection between 'feminized' domestic labour and affect has been noted, but not otherwise critiqued. In Multitude, Hardt and Negri (2004) incorporate affect and social meaning into the broader concept of "feminization of labour" (Morini 2007, n. pag.). This concept, brought about by the incorporation of women as labourers in the monetized workforce, is both broad and problematic. Through their engagement with it, they reveal a point of view in which incorporating these qualities into labour upholds a gendered discourse whereby certain types of work are presented as more masculine, while others are more feminine. While their point is to highlight the way affective labour differs from more conventional modes of production rather than to imbue affect with gendered significance, the use of gendered terminology is difficult to parse without concluding that although socially constructed, the association between affective qualities and femininity is inevitable. While it is not the aim of this chapter to tackle this injustice, failing to highlight it would be incomplete.

\section{Hang on, travelling woman: grounding taste performance and affect in a finished object}

In order to better understand how affect and taste performance can be perceived as contradictory, it is helpful to look at a specific example. The Traveling Woman shawl, pattern created by Feministy.com blogger Liz Abinante (2009), is a highly regarded and popular finished object on Ravelry. Traveling Woman illustrates how Ravelers saturate their patterns, labour and finished objects with affective and intertextual meaning, 
while simultaneously absorbing these objects, and the materials they are created from, into fashion and trend. The pattern for Travelling Woman is itself a 'hot commodity' on Ravelry. While the pattern is available for free on Liz Abinante's blog, it is also for sale through Ravelry's patterns database, where Abinante receives the majority of the profits but Ravelry reserves a small cut which goes towards site running costs. Although the pattern is available free of charge, the 7453 people who have accessed the pattern through Ravelry suggest that there is perceived value in paying for the pattern rather than getting it for free, presumably as a marker of support for the pattern creator. Already at the pattern stage, a two-fold meaning of Traveling Woman is apparent: the pattern has gained commodity status as it is a commonly sold item, and Ravelers perceive an affective benefit from paying for this commodity as they are expressing support for and appreciation of both the pattern author and Ravelry itself.

Abinante herself expresses an affective attachment to the pattern in the blog post "Traveling Woman" where it was first published on feministy.com. She writes that she named the shawl after the song "Travelling Woman" by British recording artist Bat For Lashes, and also the character Angela Montenegro from Fox Broadcasting Company's TV show Bones (feministy.com "traveling-woman"). The lyrics of Bat For Lashes' "Travelling Woman" describes "a brilliant woman with a promising future, who loses it all because she fell in love with a man who had too much potential, and not enough substance" (feministy.com "traveling-woman"). Similarly, Abinante interprets the character of Angela as "just the type who might get in a little too deep before she can find her way out". Carrying over these affective interpretations of texts from popular culture into a physical object draws attention to intertextuality as a contributing factor to "Traveling Woman"'s popularity. Furthermore, "[the] edge of the shawl represents the dangerous web of love, as well as its highs and lows" - another expression of the physical object being imbued with affective meaning. This seems a lot of backstory for a knitting pattern, but the enthusiastic response from the Ravelry community in the form of 2124 forum posts, 494 blog posts and 92 comments on the pattern itself shows that Ravelers responded to this emotional background. No doubt, the popularity of Bones, the indie appeal of Bat For Lashes and the relative simplicity of "Traveling Woman" as a knitting pattern contributed to its popularity. But the amount of care and attention Abinante 
paid to the story behind the pattern, and the effort she went through to communicate this, gives the patterns a unique flavour and imbues it with affective meaning.

However, it is not as simple as the pattern striking unique chords with Ravelers as individuals. Once a pattern becomes so ubiquitous that it verges on the memetic, it becomes difficult to distinguish affect from fashion. Are Ravelers knitting this pattern because the lyrics of "Travelling Woman" and the interpretation of Angela Montenegro resonates with them, or because doing so yields social capital in the form of demonstrating belonging through taste performance, i.e. following fashion? Is it really feasible to separate the affective and the fashionable in this case? As hip domesticity shows, the affective may be what is most talked about and celebrated within hip domestic communities, but fashion and commodity culture are still accepted and meaningful. Affect may to an extent conceal the role of commodity, but the relationship between the two is not sinister or conspiratorial. As is becoming clear through this case study, in order to understand the complexities of meaning on Ravelry it is necessary to adopt an enmeshed way of thinking where previously irreconcilable categories such as consumer culture and affective labour now overlap and flow into each other. This blending of affect with commodity is present in Ravelry's spaces, and becomes apparent through close analysis of objects such as "Traveling Woman".

\section{A critique of affect and immateriality}

So far, this chapter has drawn a distinction between immateriality and affect, using both terms deliberately in different contexts. However, in studying the labour of production in digital cultures, immateriality and affect are usually discussed in relation to each other, or even used interchangeably (see Coté and Pybus 2007, Dowling et al 2007, Shaffer 2008, Paasonen 2010, Bratich 2010, Martens 2011). Indeed, the journal Ephemera devoted its first issue of 2007 entirely to these two concepts as they are grouped together and applied in studies of new media and technocultures. The concepts are not separated, but referred to as "the immaterial and affective labour thesis" and also "immaterial and/or affective labour" (Dowling et al 2). However, it is important to note that, when speaking of technocultures and online community, the idea of affect is distinct from that of immateriality because it has a much wider potential for branching out into the offline. Affective labour, the work done to bring about an emotional 
(ex)change, can potentially be identified practically anywhere and in almost any context, as it hinges on subjective experience rather than a set of external parameters, technological or otherwise. The affective is tied to some quality or dimension not easily quantifiable or observable. While affect can be observed everywhere, within scholarly work there appears to exist a particular relationship between affect and social media, as well as the relationship between affect and domestic labour. Ravelry demonstrates the interconnections between domesticity, fibrecrafts and social media. Existing work relating to the central concepts of amateurism, social/cultural capital and domesticity, as visible on Ravelry, tends to hone in on affect as an explanation of the significance of labour taking place within social networks. These concepts are similarly tied to a particular historical context in which the domestic, affective and amateur have gradually been rendered visible and absorbed into mainstream, conspicuous activity. Both the notion of affect and this historical context will here be examined in relation to each other.

Immateriality has been utilized as a default concept for charting and explaining online labour. This is most likely because the term is to some degree flexible enough to characterise aspects of all online labour by describing the process of exchange between an individual's mind and that individual's interaction with technology. This 'immaterial' interfacing makes mental processes tangible, which in turn shapes and creates communities and cultures. However, immateriality or interfacing alone do not account for the creation and maintenance of online communities and cultures. The other dimension of this process is discussed using the idea of affect - a concept not easily defined beyond the vague notion of being something which imbues labour with social and interpersonal meaning. The slipperiness of these concepts calls into question how productive it is to separate the immaterial and affective from the wider spectrum of labour necessary for successful social media participation. As they are so broad, it seems nearly any type of labour could be reclassified as either immaterial or affective. So what work is really being done when these terms are applied? How do we account for and explain the visibility of domestic work in social media? As a site which makes the connection between the on- and offline apparent and explicit, a close look at Ravelry will provide some clues to the applicability and consequence of immaterial and affective labour. 
When applied to new media, the underlying idea of immateriality is directly tied to the use of computers: immaterial labour is a distinct type of disembodied work made both possible and necessary by our continued reliance upon ICTs, and this labour shapes and produces social, symbolic and cultural meaning online. This interpretation stems primarily from Hardt and Negri's 2004 work Multitude, as in Lazzarato's own work immaterial labour is strictly tied to the commodity: it is "the thing that lends cultural and informational content to a commodity" (1996). However, much of the literature surrounding new media and technocultures revolves not strictly around commodity or the marketplace, but social networks, interpersonal exchanges and alternative economies. Terranova (2000), for example, writes of free labour online as existing within a wider framework of gift economies emergent in the present day, not as a counter to capitalism, but as a logical "next step", a force "within late capitalism as a whole" (36) that exists neither in capitalistic symbiosis nor as a counterpoint. Accepting that Terranova has identified a nuance of labour expressed particularly clearly in technocultures, this seems to leave gaps between Lazzarato's original concept of immaterial labour and the way this labour is both made manifest and talked about within new media. If the work is not done to inject commodities with social meaning, what purpose does it serve? The key concept typically employed to fill this gap is affect. In Multitude, Hardt and Negri propose affective labour as a second type of immaterial labour, somehow both part of and distinct from the immaterial labour they define as producing "ideas, symbols, codes, texts, linguistic figures, images and other such products" (Empire 293). Affective labour, on the other hand, "produces or manipulates affects, social networks and forms of community" (Hardt and Negri in Paasonen 2010). This seems an attempt to account for the gap left between the commodity-centric original model, Hardt and Negri's redefined model in Empire, and the realities of the present-day online.

\section{Conclusion}

At times, the blurring of boundaries between affect and immateriality can stand in the way of concision and clarity. Discussing the two ideas as though they are one erases the nuance Hardt and Negri present in their work. For this reason, the terms have so far been employed as having separate but related meanings. Affect is an emotional quality brought about through and during labour; the fuzzy, hand-knitted 
object on Ravelry signifies an affective outcome, the investment of manual labour is understood as producing a subjective, emotional result with social meaning. It is therefore a quality found within labour, not a type of labour itself. Immaterial labour, on the other hand, is a step in a lengthier process where interfacing connects the on- and offline, the internal connects to the external, where there is potential for bringing about affective results. Both concepts are controversial in the sense that they are difficult to pin down and observe. Yet, they have become mainstays of scholarly work in new media.

The accumulation of social capital, defined by Bourdieu as "resources that may be called upon by the sole virtue of being one of a network of durable social relations" (quoted in Wacquant 69), plays a vital role in participation in social networks. Understanding this process of social capital accumulation as affective has both benefits and disadvantages. Hip domesticity certainly embodies elements of fashion and trend, and can therefore be interpreted as a form of commodity culture as well as a way of documenting affect. On the one hand, the affective interpretation accounts for the historicity of women's work and domestic labour, and suggests that hip domesticity is not so different from the historical context from which it has emerged, unlike new domesticity which proposes an ideological break from the past. On the other hand, affect is a slippery concept with meanings difficult to pin down, and the notion is further complicated by its presumed removal from the commercial. Affect is typically associated with non-commercial space, and in networked environments, the commercial and noncommercial are rarely discussed as though they overlap (Humphreys "The economies within an online social network market" 4). Yet, both are present on Ravelry, although it seems the affective dimension of craft-work is at times exaggerated in a manner which to a certain extent masks the presence of commodity culture. This is seen in the community's response to the story behind the Traveling Woman shawl. Here, "labours of love" are directly tied to the accumulation of social capital and the strengthening of social ties. The more offline, manual work a Raveler does, the more there is for documenting and sharing online, hence the greater the chance of gaining social status. This seems both a curious parallel to and a contrast to Cowan's "senseless tyranny of spotless shirts and immaculate floors" (216): the more conspicuous the domestic efforts of the housewife, the greater the kudos. The more conspicuous the offline knitting 
efforts of the Raveler, the greater the online social capital. Yet, "Traveling Woman" shows a series of affective exchanges taking place through and surrounding a commodity object. This complicates the distinction between affect and commerce, and suggests the need for an analytical position where these two categories can be intertwined. 


\section{Chapter 4 - Crafty commodities: buying, selling and knitting oneself into being}

\section{Introduction}

Rather than adhering to existing notions of commercial and non-commercial networked space, Ravelry offers a sophisticated example of the coming together of different types of space in a way which forms a social whole (Humphreys "Economies" 237). Ravelry's content and structure have for the purpose of analysis been divided into three distinct spaces: social space, labour space and marketplace. While social space and labour space have already been discussed, the aim of this chapter is to evaluate the role of commodity and marketplace within the Ravelry community, and discuss the ramifications of incorporating commerce into this specialized network's social reality. Handicrafts commodities are important to Ravelers. Buying, selling and displaying ownership of commodities form part of a Raveler's social life within the network. This contradicts a reading of "new" domesticity as resisting commoditization as per Emily Matchar's mission statement in her blog New Domesticity, and supports a reading of hip domesticity as containing aspects of trend and consumer culture. The relationship between the commercial, the social and the affective that is visible on Ravelry supports a reading of this social network as a reality where all three spaces are enmeshed, where all spaces carry prestige and significance, without any one being dominant. The previous chapter argued that reading domesticity as purely affective is problematic as hip domesticity has become absorbed into fashion. It therefore operates on a level which is not just affective, but also trendy and popular. This trendiness challenges the understanding of handicrafts as affective, "pre-capitalist" (Bratich 316) activities. Domesticity as fashion also calls into question whether or not "new" domesticity really functions as the quasi-feminist social movement geared towards challenging the mass consumption and unethical business practices Jean Railla and Shannon Hayes respectively rally against in Get Crafty: Hip Home Ec (2004) ad Radical Homemakers: Reclaiming Domesticity from a Consumer Culture (2010).

The presence of a commercial marketplace on Ravelry adds a layer of social meaning to commodity ownership because it is incorporated into the site's structure 
through both its social space and marketplace. Rather than offering 'resistance themes' (Minahan and Cox 11) and denouncing commodity culture as running counter to hip domesticity, Ravelry incorporates it. Supplying evidence of commodity consumption contributes to the Raveler's textual performance, and engaging with others over commodity choices through comments, reviews and ratings is common throughout the site's social space. This means that Ravelry requires its members to buy, as well as knit and write, themselves "into being" (Sundén 88). Ravelry offers many avenues for members to display their knitting commodities, and the most prominent of these avenues is the "stash". The stash is an area in "My Notebook" where Ravelers catalogue and display yarns and other knitting materials they own. It mirrors 'projects' in form and structure, but rather than displaying objects Ravelers have produced, it displays objects Ravelers own. Production and ownership therefore both contribute to how Ravelers build their identities through the process of recording and cataloguing. The display of commodity ownership is viewed as an important part of Ravelry's function along with documenting labour and interacting with others, and records of ownership in 'stash' can be just as detailed as records of labour in 'projects'. Ravelers therefore represent themselves not only through their offline, manual production, but through the commodity acquisition underlying this labour.

The idea of knitting and homemade crafts as existing primarily on an affective level is often repeated in scholarly work as well as among knitters themselves, leaving little space for discussions of commodity exchange. Significance is attached to the warm, fuzzy emotions which arise out of producing and gifting knitted objects, and knitting is interpreted as care-work first, manual labour second. Historically, knitting and other forms of craft have been "performed as a gift-giving practice and as a form of care for others" (Bratich 306) as well as being useful for the production of everyday necessities. However, despite the glorification of affective labour within modern-day hip domesticity, the presence of capitalist ideology and a thriving marketplace cannot be ruled out as important to the social reality that is visible on Ravelry. In fact, much of Ravelry's "affective labour" rests on a foundation of commodity exchange. This commodity exchange is in turn veiled by notions of affect, as though the affective outcome of documenting and gifting a hand-made object negates or disguises the commodities it was created from. For example: before a gift-object can be made, 
materials may need to be purchased. These purchases are governed by intricate and subjective guidelines, where a finished object's affective potential is influenced by the material's status as a commodity, such as the "Traveling Woman" shawl discussed in the previous chapter. These materials are yoked to affect in a complex relationship in which a finished object's "affectiveness" is the sum of both the commodities chosen and the labour carried out. The perceived value and desirability of materials, then, contribute to the finished object's status. However, there seems to be a reluctance to acknowledge the significance of commodity exchange in the world of handicrafts, in both theory and practice (Bratich, Minahan and Cox). As already established, the status of the finished object denominates its value as an entry token into Ravelry's social world. The trendiness and social relevance of crafts like knitting hinge on an affective ideal, but also require consumer savvy. This commercial aspect seems at times overlooked or glossed over in academic work on fibrecrafts and technocultures. Holding crafts up to an affective ideal ignores a wider social context where what people spend their money on and what they own matters. As Tiziana Terranova observes, people are accustomed to being addressed as "consumers of meaningful commodities" in postFordist capitalist society (Free Labor: Producing Culture for the Digital Economy 37). As Liu observes, "possessions and consumptive choices [have been] brought into the fold of identity (252). Ravelry is not immune to this commodity culture - in fact, the deeper one delves into the readings of affective relationships on the site, the more the microtransactions and commercial imperatives supporting these relationships become visible. On Ravelry, commodification plays a role in the representation of self, and is one of many fragments required for knitting oneself into being. The role of commodity culture becomes evident in records of labour in "My Notebook", as finished objects are made up of existing commodities such as yarns and patterns. These underlying commodities contribute to the shaping of identities produced when Ravelry's social spaces and labour spaces intersect with the marketplace.

This means that Ravelry is neither a fully commercial nor fully non-commercial networked environment. As Sal Humphreys has pointed out, networked environments have a tendency to be interpreted in a binary fashion as either market or non-market ("The economies within an online social network market" 4). Similarly, handicrafts networks online have often been framed as pseudo-activist communities resistant to 
"mainstream" ideologies such as capitalism, embracing instead cyberfeminism, sustainability and alternative economies (Bratich and Brush 2007, Bratich 2010, Minahan and Cox 2007). Through its intertwining of the social with the commoditised, Ravelry complicates a binary reading of its networked environment as either commercial or non-commercial. Speaking to social networks in general, Humphreys observes that "[social network markets] operate across a diverse range of economies from the commercial to the affective" (1). On Ravelry these economies overlap, intermingle and enhance each other. Ravelry's social economy seems tied to its financial economy, but this relationship is neither straightforward nor immediately apparent. Social economy is the means through which social capital is exchanged, gained and maintained, and as stated previously the motivation to build and strengthen social capital is a mainstay of social networks (Ellison, Steinfield and Lampe "The Benefits of Facebook "Friends": Social Capital and College Students" 2007). Ellison et al. observe that the flow of social capital is not limited to the online world; participating in a social network can generate and strengthen social ties both on- and offline (1145). In this sense, Ravelry is similar to Ellison, Steinfield and Lampe's observations about Facebook users, but Ravelry offers one significant point of difference in the way labour itself is utilized as a means of gaining social capital. Ravelry's domestic labour, and the affective, intangible qualities surrounding it, rests on a foundation where buying and selling commodities to a certain extent determine the labour carried out. Being seduced by affective ideals without accounting for trend comes at the expensive of a fuller, more nuanced analysis of Ravelry's enmeshed spaces. Ravelry promises emotion, but delivers fashion. Without being a fully commercial operation, the site is undeniably tied to commerce. These dynamics help shape the relationships and norms which are established within the site.

\section{Recapping Ravelry's spaces of interaction}

Ravelry's spaces became evident in the reading of the site as a textual object, though they need not be present in the minds of Ravelers themselves. As such, they are a heuristic device. Categorizing the site into spaces allows a teasing out of its distinctiveness, illuminating its points of departure from other SNS while maintaining its anchoring within social media as a whole. The first space, social space, accounts for all the different ways Ravelers directly interact with each other. This is the space where 
interpersonal written communication occurs, and where a Raveler can write messages straight to the network. Social space includes Ravelry's messaging systems, commenting/status update functions and above all, its active and involved forum. These online hang-outs are the backbone of social media, and it would be impossible to point to a social network without also identifying its social space. However, this social space did not spring forth fully formed and independent. On Ravelry, social space relies on its entanglement with other types of space and other forms of user activity.

The second space is called labour space and as discussed in the previous chapter, this is where Ravelry's distinctiveness becomes apparent. In their labour space, Ravelers photograph, catalogue and otherwise document the work they have carried out offline. This process of categorization and classification is in itself a form of labour, creating a 'double whammy' of work perceived as necessary for successful participation in the social network. This emphasis on carrying out and documenting labour is intrinsic to the site's architecture. The Raveler's Notebook, in which records of labour are stored, has to a certain extent taken over the role of the traditional SNS profile, although more conventional profiles are also present on the site. The importance of the notebook is emphasized through the number of connections made to it via hyperlinks throughout the site. This contrasts with the actual user profile, which is barely linked to at all. A Raveler without anything in her notebook could still be participating in the social space through forums, commenting on other people's projects and such, but not complying with the expectation of labour-documenting comes at the cost of social capital. This is visible in the forums, where members who do not fully adhere to community norms often struggle to be 'heard' and receive responses to their posts. For example, new members who have not yet built up records of labour in "My Notebook", or do not have a profile picture which shows their faces and/or a finished object, or lack any history of posting in the forums often struggle to receive responses when they post asking for help. This is particularly evident on the patterns board, one of Ravelry's 6 main 'boards' or forum sections (Ravelry.com "discuss/patterns"). New members unfamiliar with the significance of not only speaking of, but also showing evidence of one's work will often have their requests for pattern help met with counter-requests for pictures, hyperlinks or other forms of evidence of manual production before an answer to their question is given. 
At the opposite end of the spectrum there are a number of Ravelers who meticulously update their notebooks with current projects, finished objects and so on, without actively participating in the social space. They are connected to others automatically via records of similar completed projects, but were it not for their labour records they would not be visible on the site at all. The notebook can therefore act as a stand-alone tool for knitters who want to archive their labour for their own benefit, motivated by something other than "knitting themselves into being" for the social network. Wanting to use "My Notebook" without participating in Ravelry's social life reveals the affective dimension of this type of labour. While people can maximise the usefulness of their Notebooks by utilizing it to strengthen social ties, the Notebooks contain a record of affect no matter how much it is shared. How many knitters use "My Notebook" without making much use of social space is unknown - this project identified several, but finding people who do not wish to socialize is inherently problematic. However, the fact that labour space can be used to different ends demonstrates that it is identifiably different from other types of activity on the site.

The final space, and the focal point of this chapter, is the marketplace. Commercial activity such as buying and selling is present throughout Ravelry. From the welcome screen to the depths of specialized searches in the patterns database, Ravelers are encouraged to purchase commodities. Ravelry is commercially operated and funded through advertising, commissions and its own mini-mart, but these are the more straight-forward areas of Ravelry's marketplace similar to those found in other advertising-funded SNS. Rather than looking at, for example, the mini-mart, advertising, or other commercial ventures necessary for the upkeep of the site, this analysis will focus on the way Ravelers themselves interact with commodities, and by extension, how they interact with each other through these commodities.

What becomes clear through close analysis of relevant areas of the site, particularly those relating to yarn and yarn ownership, is the impression of ingrained commodity exchange where Ravelers themselves do not necessarily draw distinctions between exchanging commodities and creating objects with affective value. In fact, the commodity-acquisition underlying the creation of a finished object seems important to the object's status. A finished object's status can be enhanced when it is created from exclusive commodities, just as it can be "talked down" if it has been created from less 
desirable, everyday materials. Evidence of Ravelers buying, trading and gifting commodities amongst themselves is found virtually everywhere on the site: patterns can be browsed using search parameters only returning results which are pay-toaccess, local yarn stores (a 'LYS' in Ravelry parlance) can be located based on a Raveler's location in a single click, details surrounding projects in "My Notebook" are autocompleted with links to stores materials were purchased from, websites of yarn manufacturers, and so on. Ravelers can also offer their own wares for sale by clicking through simple templates. Yarns in particular are commonly sold and traded amongst Ravelers without any third-party input. This can be thought of as a type of user-driven marketplace: it is not directly controlled by external commercial operations such as yarn and knitting needle manufacturers, but still governed by norms established by Ravelry's user base. This is consistent with Carolyn Wei's observations about formations of norms in knitting communities (2004, n. pag.): top-down guidelines can be implemented by any perceived authority, but the community will still shape actual practice in ways they see fit. These norms build on existing and familiar ideals of the social value of commodities and rewarding entrepreneurship (Humphreys "Economies within an online social network market" 12,18) while remaining anchored to the historicity of crafts and domestic labour. Therefore, the financial is inextricably tied to the social and affective. Of particular interest is how varying forms of economy play into the accumulation of social capital, and how this in turn shapes the user-driven marketplace as a whole. By studying Ravelry as a trifecta of overlapping or interlinked spaces, it is possible to access and engage with the complexity present in the way its commercial and non-commercial spaces intersect. This overlapping of affect and commodity is an area where few scholars have reached any agreement, or have produced similar arguments. Ravelry produces an opportunity for better understanding how these spaces work together in a manner which produces social meaning for its members.

\section{What's in your stash? Ravelry's user-driven yarn marketplace and discourses of exclusivity}

So where can we most readily observe the intersection between the commercial and the non-commercial? The 'stash' tab in "My Notebook" (see fig. 2) is where Ravelers display their ownership of raw material, i.e. their yarn. ${ }^{13}$ Previously, this project has 
looked at the 'projects' area of "My Notebook” where Ravelers record their labour. The 'projects' and 'stash' tabs are markedly different in purpose. While 'projects' records and displays finished objects (the results of offline labour) without immediate links to the marketplace, 'stash' is explicitly commercial with its emphasis on brands, names of manufacturers and local yarn stores on its front page. It follows a visual layout similar to that of 'projects' where finished objects are displayed, but emphasises the stash entry's status as commodity rather than affective object. "My Notebook" encourages recoding, ranking and sharing as many details as possible about any given yarn, including how this was acquired (gift, purchase, handspun, trade) and whether or not the yarn is available for further sale or trade. This mirrors the way "My Notebook" encourages the record of as many details as possible about a finished object within 'projects'. Within "My Notebook", 'projects' and 'stash' complement each other, but are not necessarily co-dependent as

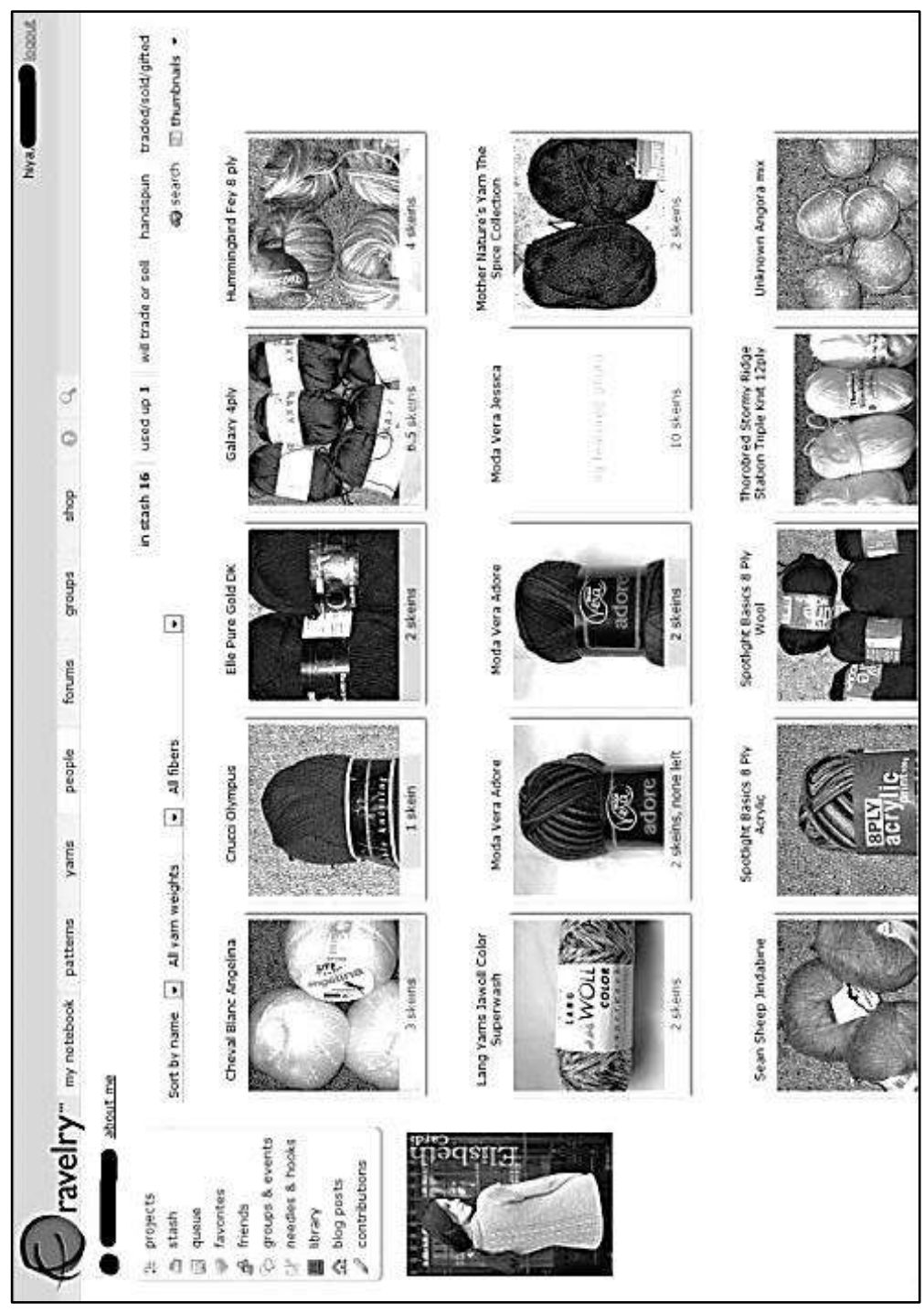

Figure 2 - A Raveler's stash (used with permission) no information crosses over from one to the other unless the Raveler specifies that this should happen.

An important point of difference from 'projects' is that 'stash' contains within its layout means for offering yarn back to other Ravelers through processes of sale or exchange. Finished objects in 'projects' cannot be sold in the same fashion. Ravelers 
simply need to pick the correct pre-set value from a template according to the yarn's status (is it available for sale, trade or gift exchange?) in order for it to be placed "on the market". Any member can look up any other member's stash via hyperlinks from their Notebooks, and read individual entries such as what store a particular yarn was purchased from, how the Raveler rates the yarn (in stars out of 5), and whether anyone else has commented on or 'favourited' this particular entry in a Raveler's stash. Any stash record in "My Notebook" is cross-referenced with Ravelry's yarn database, which simplifies the process of recording by auto-completing known information about particular brands and types of yarn. It is common to also upload pictures of the materials in ball, hank or skein form, and these photos are usually taken by Ravelers rather than, for example, copied from the yarn manufacturer's website, indicating that Ravelers prefer to create and present their own visual meanings, despite the convenience of readily available stock photos. This suggests that creating and presenting visual meanings of one's own carries affective significance; it shows a further labour investment into the 'stash' entry and shows that the Raveler took this extra step in order to further personalize the record.

As alluded to previously, the hierarchy of commodity found within Ravelry is unique to the social network itself. The opinions and recommendations of other Ravelers carry more weight than the more "derivative, commercial" mechanisms of advertising which put Ravelers in direct contact with retailers (Humphreys 7), although text-link ads to businesses, typically local yarn stores (including their location and opening hours), are automatically inserted into "My Notebook" entries as per Ravelry's advertising guidelines (Ravelry Advertising and Pricing 2). Ravelers have come up with their own set of criteria for judging the exclusivity and desirability of any particular yarn. This is illustrated partly through the rating system, where yarn types from individual brands are given stars out of 5 based on the cumulative ratings of the community. But it is also illustrated through the opportunity Ravelers have to comment directly on a yarn, leaving micro-reviews, warnings, technical information such as yardage and weight, or anything else deemed beneficial for the community. Yarns appear to be rated based on a complicated set of assumptions about quality, ecofriendliness and ethics, balanced with cost, ease of access and variety. One might think the higher a yarn is rated, the more commonly it is used, but this is not entirely the case. 
One might also think that ratings out of 5 would distribute in a curved fashion with the majority of ratings falling towards the middle (for example between a 3 and a 4 ) and the highest and lowest ratings reserved for the exceptional and the abysmal, but this is also not true. At the time of writing (10 September 2012), there are 40,325 yarns which have been rated by users in the Ravelry database. 18,386 of these yarns have a top rating (a solid 5 stars), while the remaining 21,939 yarns are rated between 1 and 4 . Combined, yarns with a 4 to 5 star rating make up the vast majority $(35,069)$ of yarns in the database. With only 5251 yarns receiving a rating of average or below, the higher star values would appear almost meaningless to an outsider. This indicates that the threshold for receiving a high rating is low, and that rating a yarn highly does not necessarily hinge on actual expense, exclusivity or exceptional quality, but on other qualities deemed significant by the Ravelry community.

With so many yarns receiving 4 and 5 stars, one would also assume that there would be little reason to ever knit with anything with a lower rating. Yet, as the $2^{\text {nd }}$ most commonly used yarn on Ravelry with 87,655 projects recorded, Red Heart's Super Saver Solids (100\% acrylic) only has a rating of 3.27. The majority of the 116 comments left on this yarn are neutral to negative, citing a plastic feel, scratchiness, discomfort and lack of versatility as its most limiting factors. The yarn is redeemed by its low cost, variety of colours and general resilience. One commenter summarizes the overall attitude expressed in the majority of comments perfectly: "It's cheap, and I can afford it, but I HATE this yarn" (Ravelry.com "red-heart-super-saver-solids/comments"). In other words, this Raveler is willing to work with Red Heart Super Saver Solids in order to pursue her hobby and interact with the social network. Yet, she expresses dismay at needing to use this particular yarn, as though apologizing for using it is necessary, even though the affordability and availability of Super Saver Solids allows her to continue knitting and posting content on Ravelry. This contradictory language is commonly adopted by the thousands of Ravelers who have used Red Heart Super Saver, as witnessed in these comments. They are aware of its dubious social standing (giving away an object knitted with Red Heart is often accompanied by a sort of apology in the finished object's entry in "My Notebook"); its low cost and unpleasant attributes are explained and justified as acceptable only in certain situations, like "those small, odd ends project where you simply want a regular, not too expensive, acrylic yarn" where "it 
does have its place, such as toys and other items where destruction level needs to be high, but I'll never make another garment from it" (Ravelry.com "red-heart-super-saversolids/comments").

If Red Heart Super Saver is so substandard, the reason for its ubiquity is surely its availability and low cost. Using it may devalue the affective potential of the finished object and its potential for generating social capital, as giving a gift made of Red Heart does not hold the same connotations as gifting an object made of Ravelry's third most widely used yarn, Malabrigo Yarn's Merino Worsted. Unlike the apologetic language used to describe Red Heart, what is evident from the user comments on Malabrigo Merino Worsted is the perception that this yarn is exclusive, high quality and capable of producing treasured finished objects that possess an extra affective "cuddle-factor" (Ravelry.com "malabrigo-yarn-merino-worsted/comments"). It should be noted that Malabrigo is a mass manufacturer and its yarns are easily found throughout the United States (where most of Ravelry's users are based), and it is therefore as readily available as Red Heart's Super Saver Solids. Yet, the perception that this yarn is more exclusive and possessing higher potential for social and affective reward is clear in the way it is talked about. Its softness and beauty are lauded, user comments include emotive language like "I am a believer" and "It's easy to understand all the people I've seen saying they want to marry this yarn!" (Ravelry.com "malabrigo-yarn-merinoworsted/comments"). A few lone voices of dissent on the 6 pages of comments make apologies for their lack of enthusiasm, citing poor quality spinning, inconsistency between batches and the yarn's tendency not to hold up to regular wear as factors which should detract from the yarn's overall popularity. But even in their criticisms they allow for the possibility of simply having used the yarn in the wrong context, promising to try it again or blaming their own skill level for the failures of this yarn. They acknowledge the social standing of the Malabrigo brand, and assume their own failure is at least partially responsible for their misadventure with Merino Worsted. This seems to replicate the way brands are talked about and compared everywhere. Ravelers may have come up with their own rules for judging quality, but the discourse of exclusivity they apply to yarns such as Malabrigo Merino Worsted is an 'added value' invented by the community itself, rather than something which is imposed upon the 
consumer by the manufacturers. Ravelers may produce their own meanings, but the dynamics of commodity-acquisition mirror consumer culture everywhere.

The brand culture prevalent in user stashes may at first glance seem a jarring contrast to the more feel-good affective fuzziness encountered in records of finished objects. It certainly makes clear the marriage of commercialism and the social network. However, it is important to remember that just as the ideals of the affective are problematized by the absorption of hip domesticity into fashion, so is the commercial problematized by its incorporation into the social structure on Ravelry. Rather than looking at Ravelry's marketplace as a commercialization of social interaction in which coveted commodity equates popularity, it is more productive to approach it as an opportunity for exploring the link between relationship-building and different forms of capital. Owning the "right" yarns and holding the "right" opinions according to the community's standards may contribute towards a Raveler's social capital, but displaying ownership of popular commodities is on its own unlikely to yield any results unless the Raveler participates across the full range of Ravelry's spaces. The stash highlights the "unruly behaviour of productive users consuming and producing across a range of motivations and to varying ends" (Humphreys 2009 6). Yarns without finished objects equate commodities without affect; their social meaning is intertwined and cannot easily be plied apart.

\section{The micro-economies of Ravelry: enmeshing handicrafts and capital}

Unsurprisingly, it is primarily yarn that is advertised, bought, sold and rated within Ravelry's user-driven marketplace where Ravelers buy, trade and sell amongst themselves. However, other materials like unspun fibres, knitting needles/crochet hooks, beads and other add-ons are also bought and sold on a smaller scale. Buying and selling among members occur through varied and sometimes innovative channels. Exchanges take place informally through forum posts where requests for "reimbursements" are common and set prices are not, formally through a Raveler's own shop or stash (linked to from her Notebook), or privately through direct messaging between members. In theory, transactions should take place within Ravelry's own setup for buying and selling as this allows Ravelry to receive a small commission, contributing to the site's running costs. In practice, small transactions take place 
through many different channels, where members often use the language of trading and exchanging gifts without necessarily drawing attention to potential profit-making, even though money may often change hands. As mentioned previously, the relationship between profit-making, affect and online handicrafts communities is somewhat contentious. Some, like scholar Jack Bratich, hold crafting and online crafts communities up to a standard where activities such as knitting embody a tradition of gendered carework which pre-dates capitalism, and now exists alongside it rather than being embroiled within commodity culture ("The Digital Touch" 2010). In other words, capital is here seen as something separable from the affective. It is possible that this view of the handicrafts revival as a re-emergence of 'non-capitalist' affective work stems from a desire for self-valorisation in a manner which does not depend upon commodity culture; affective labour is the focal point and finished objects are tokens exchanged in a gift economy, not objects produced out of commodities and retaining some degree of commoditized value. Others, like Sal Humphreys, look at the enmeshing of commodity, affective labour and social capital as forming a whole necessary for the success of a network such as Ravelry. Commodity and affect are not necessarily separable, and they are not mutually exclusive. Through the analysis of its marketplace, Ravelry seems to support this second reading of the relationship between affect, commodity and social capital: small commodity exchanges take place continuously and commodity ownership is readily displayed, but this does not 'cancel out' the affective value of finished objects as tokens of care-work, warmth and gift-giving. Ravelry is not a site primarily about buying and selling crafts commodities, nor is it a site primarily about displaying evidence of affect. Both are equally important; they are intertwined through the site's architecture suggesting that their significance in building a social reality is evenly matched.

Bratich and Humphreys both address why manual, offline labour has become socially meaningful online, though their approaches and conclusions differ. Both look at how the self is brought into being in a social network. Sal Humphreys connects the dots between economies and social networks in a way which highlights the mutually dependent relationship between the social and the financial. Rather than extracting capital exchanges from Ravelry's tangled spaces, Humphreys approaches the financial transactions present on the site as yet another important facet of a specialized, well- 
functioning SNS. She calls on Ravelry to be the object of her case study due to Ravelry's "complex blend of users, economies and motivations" ("Economies" 2) which sheds light on its specificity as a stand-alone resource for a specialized audience, but also makes it easy to see parallels and similarities between Ravelry and other social networks. Humphreys' contribution centres on the difficulty of drawing a meaningful distinction between the commercial and the non-commercial within social network dynamics using existing theory, as compartmentalizing different modes of production fails to explain the number of ways these different economies converge. She claims that through sites like Ravelry, "we are seeing in the development of social network markets the beginning of the crumbling of the distinction between commercial and non-commercial" ("Economies" 5). Humphreys focuses primarily on the micro-transactions which take place between Ravelers and Ravelry's interpersonal commerce, and uses this in order to challenge existing, compartmentalized theory in which sites are either commercial or non-commercial. But it is not only within Ravelry's commercial arenas that categories previously thought separate and distinguishable become enmeshed. The same is true of labour: through the need for manual labour as a necessity for participation in its social network, Ravelry grounds the work of its users in tangible materiality. This exemplifies the shortcomings in reading online labour as immaterial. But it also adheres to conventions of social media 'work' through its use of favouriting systems, comments, 'friending' and carrying out other writing and clicking tasks. Ravelry's combination of being an exclusive specialized site while at the same time being easily recognized as a social network, following established social network logic, offers fertile ground for theorising the relationships between affect, social capital and labour.

As a point of contrast, Jack Bratich approaches handicrafts and affective labour from an autonomist social theory perspective. In his 2010 article "The digital touch: Craftwork as immaterial labour and ontological accumulation", he argues that the modernday revival of crafts and other types of DIY is both antagonistic and historically rooted, responding to and pushing against capitalist valorisation of labour above the valorisation of the self. His focus is placed on the concept of crafting more than the actualities of knitting and participating in online crafts communities. Rather than looking at the in-depth detail of any particular type of handicrafts labour, Bratich speaks of crafting as a singular concept, surmising that these crafting activities have enough in 
common that they can be addressed as a whole. He sees the resurgence, or what he describes as 're-composition' of handicrafts as a return to what he refers to as "precapitalist" activity.

In this re-composition, perhaps, we can include a type of repetition: a refurbishing, a restoration, a renovation, among capitalism's ruins. Amidst these ruins, the old cracks begin to expand; the occulted circles reach out beyond the sewing room to weave their fabric again. This recomposition sees a return of those technics and knowledges that have comprised biopower, the power of life itself. (Ephemera 316)

Bratich here uses a very material example in order to demonstrate high hopes for the restorative possibilities of crafting, particularly through their presence online. He sees the marriage of handicrafts and digital technology as inevitable and complementary (307), and suggests that crafting had a linguistic presence within computer technology long before anything like social media existed. Through words that cross over between the manual and the virtual (such as digital, the Web, the Net, fibre, and so on) Bratich uses this "linguistic trick" (303) to tie the online and the offline together. This trick is perhaps a little overstretched - he cites Sadie Plant's suggestion that the binary code of computer programming references knit and purl (put in writing as a 1 and a 0 , but signifying the binary states of on and off) (305). This could have provided an elegant segue into the fluidity of the on- and offline in crafting on Ravelry, were it not for the fact that $1 / 0$ suggests a diametric opposite or binary (on/off), whereas a purl stitch is simply a knit stitch inside-out and vice versa. Knit/purl comprise the same stitch, appearing as a knit or a purl depending on which side of the knitted work you look at. In certain other languages, 'knit' and 'purl' do not have separate words, but differing prefixes or denotations indicating which side the stitch should be knitted from. In Norwegian, for example, a knitted or purled stitch is called a 'maske' (stitch), whereas 'rett' (meaning straight, right, or in this case forwards) and 'vrang' (meaning inside-out) denote how the 'maske' is to be knitted. The Japanese word 'amimono' encompasses all crafts made from yarn or string (Nargi 7), and while most commonly used to refer to knitting, also refers to mesh-making in general. These other 'linguistic tricks' shatter the binary simile in the context of actual handicrafts, as opposed to computing. Knitting is in itself a process of making mesh; the tangled web of relations on Ravelry is comparable to the material outcome of knitting. 
Bratich goes on to suggest that "fabriculture has occurred alongside of digital, virtual culture" but in a relationship where fabriculture acts at different moments as “complement, opposition, or antagonist" (305). This complexity mirrors some of Sal Humphreys' observations about the vexed state of Ravelry (and other knitting communities by extension) not fitting neatly within existing categories of commercial and non-commercial, where the social, affective and financial all come together in a fine mesh. But Bratich's approach then slots knitting and other handicrafts into a meme of resistance, where the affective and the social trump commercial imperatives. He draws on the history of women's knitting and sewing groups as breeding grounds for early feminist movements despite being dismissed as sites of insignificant, non-valorised “women's work" (308), and sees a parallel with online knitting communities today. Furthermore, he sees crafting as "untied from capitalist valorisation" (309) by virtue of its affective qualities, tied to the historicity of women's domestic space and care-work which existed outside the capitalist world of waged employment. He views the giftgiving aspects of crafts communities (including mentorship, community-building and exchanges of knowledge as well as exchanges of material gifts) as a resurgence of a gift economy encoding "a desire for the pre-capitalist form of production, for the "personal touch'." (309). He argues that this desire, and crafting practices in general, never truly left and have retained a presence throughout modern history, but courtesy of its marriage to digital technology it is now coming to the forefront.

Rather than romanticising crafting as a return to an idealized former society, Bratich acknowledges that such revivals are a "mixed bag" (310), not a simple nostalgic longing for the past. Certainly, there is little evidence among the finished objects displayed on Ravelry that their creators knitted them because of a desire to return to some simpler, less consumerist past. But there is plenty of evidence that the 'personal touch' is important. Nonetheless, while the personalized and unique is no doubt important as it is imbued with affect, it is difficult to claim that Ravelry offers much evidence of resistance against, for example, mass production. One-of-a-kind finished objects are often made with mass-produced, ethically and environmentally dubious yarn, marrying the uniquely affective to the readily available and commercial. Those who spin their own yarn are, through Ravelry's very architecture, required to present themselves as entrepreneurs if they wish to share their homespun goods with the site's 
marketplace. Indeed, the absorption of crafts into hip domesticity as a type of fashion complicates the idea of crafting as a resurgence of "value-production outside of the circuits of capitalist capture" (Bratich 313) and a longing for a different type of economy, where crafting itself is a source of self-valorisation and biopower (what Bratich calls "ontological accumulation"). There is no doubt that the historicity of women's domestic labour contributes to the value-production which takes place through Ravelry. It is one of many motivations underlying the act of knitting oneself into being, but extending this into more metaphysical claims about ontological accumulation seems at once problematic and hard to refute. Bratich seems to assume a known motive which knitters share, a tacit agreement about just what doing these types of crafts means. As Ravelry exemplifies, the way meaning is expressed is so intricate and varied that it becomes difficult to support claims that Ravelers all, on some fundamental level, share the same motive.

There is great value, however, in understanding the crafts "revival" not as a true revival, but as the resurgence of an undercurrent of affective material production which never truly went away, which is one of Bratich's key contributions. It is in other ways difficult to reconcile some of his observations about the self-valorising and resistive nature of crafting with the actualities on Ravelry. His argument seems greatly influenced by the sources he studied, which were weighted towards the more political arm of knitting communities. Rather than applying his argument to a range of hip domestic websites and social networks, he leans towards what he, along with Heidi Brush in their 2007 conference paper "Craftivity Narratives", calls "craftivism". While he cites a range of texts and communities (from the purely commercial Martha Stewart to the Anarchist Knitting Circle), his arguments seem to bear most relevance towards the "anti-capitalist and anti-authoritarian craftivist" (304) end of the spectrum, where the act of knitting itself takes on sociopolitical meaning greatly important to its members. These communities are indeed devoted most attention in his own publication and in the collaboration with Brush. The scope of this study therefore seems narrower than the argument might suggest. This stretches the ontological accumulation argument a little thin, as the complexities of Ravelry's intersecting spaces do not seem fully accounted for using such broad strokes. In the context of Ravelry, Bratich's theory of ontological accumulation is yet another piece of a much larger puzzle. 


\section{The cloak of affect: Yarnies and enforced entrepreneurship}

While Ravelry's spaces typically intersect and blend in a seamless fashion, there is some evidence indicating that commercialism is at times incorporated above and beyond what community norms otherwise imply. A founding principle in this study is that through the process of documenting and sharing their 'finished objects', Ravelers participate in a specific type of exchange of social capital where more labour equals more credit, but an overly commercial presence detracts from social capital. Yet, there are instances where a commercial presence is necessary before members can even participate fully on the site. This is true in the case of "yarnies": Ravelry's dyers, spinners and painters. Handspun, hand-dyed or painted yarns cannot be sold in the same manner as commercial yarns, which are readily bought and sold via the 'stash' page in any Raveler's "Notebook". In fact, if one puts down a yarn as handspun when uploading a record to 'stash', this yarn will automatically be displayed as not for sale, and this default value cannot be changed. Dyers, spinners and painters who want to share their creations on Ravelry must set up business-like profiles on invitation from Ravelry, and this gives them the recognized status of "yarnie" - a label visible to others in their user profile and their notebook. Ravelers who want to share their homespun yarn without creating records of this yarn for the database cannot do so unless it is a private arrangement with other members, they must become yarnies with a business profile first. Once Ravelry has approved the yarnie status, the Raveler can then upload records of their own handcrafted yarn. This enforces a type of entrepreneurship and blurs the lines between a commercial operation and a homemade undertaking, reflecting the observations about the breaking down of barriers between producer and consumer, professional and amateur so often referred to in new media literature (see Paasonen 2010, Humphreys 2008 and 2009, Ross 2009, Terranova 2000). An analysis of a cross-section of yarnie profiles reveals that while most have set up e-businesses to sell their yarn, a significant minority use the yarnie section only to display yarn they have made for their own use, paying lip service to e-commerce with vague promises of perhaps making available anything they cannot use up themselves.

The existence of yarnies highlights an inconsistency in Ravelry's own view of the commercial vs. the non-commercial. If you hand-make your own yarn, and you wish to sell this yarn to the community, you must present yourself as a business person. 
However, if you own yarn you have not made yourself, and you wish to sell this yarn to others, you are just another Raveler offering to share your stash for a small profit. This inconsistency could be motivated, in part, by the community's own desire to promote and support alternative forms of business, where small, semi-professional operations could so easily be drowned out by the world's Malabrigos and Red Hearts due to the general availability and lower cost of these name brands.

Ravelry actively encourages its members to advertise their own products and yarn stores through the site's self-service system where Ravelers can set up their own advertiser accounts, reserve ad space and pay via ordinary online transactions such as PayPal or credit card. Unlike many other social networks which allow advertising, Ravelry keeps its ads strictly fibre-related and does not allow any non-fibre products or services to be advertised ("Ravelry Advertising and Pricing" 1). In its policy material, Ravelry claims that for this reason, it "may be the only website around on which our members ask for more ads!" ("Ravelry Advertising and Pricing” 1). Information about advertising policies is also transparent, as it is accessible to any Raveler whether they have an advertiser account or not. This insistence upon keeping the means of advertising easily accessible to ordinary members and the ads relevant to the community's focus supports the idea that Ravelers respond positively to supporting alternative, small-scale businesses. However, in the case of yarnies, the insistence on building up and supporting small businesses limits these Ravelers' autonomy by enforcing commerce, something which is contentious within the community. For one thing, the nickname "yarnie" is applied across knitting communities to anyone with an affinity for yarn of all types, and does not strictly refer to someone who makes and sells her own yarn. Interestingly, in the ideology of the site itself yarnies are considered "brands". This is expressed in site architecture where the hyperlinks to any yarnie's profile/store looks like this: Ravelry.com/yarns/brands/yarnexplosion ${ }^{14}$ where the name after /brands/ is the name of the yarnie. This branding is tell-tale: Ravelry pits its own yarnies against established commercial brands like Malabrigo (see Ravelry.com/yarns/brands/malabrigo-yarn), thereby reinforcing a conventional commercial dynamic of brand competition. However, this also indicates a level of support for the yarnies, in that there is no perceived need to separate them from the mass-producers and large-scale professionals. 
However, such overtly commercial imperatives are often hidden under a cloak of affect. Throughout the site, and particularly in those areas relating to the buying and selling of homespun, handpainted yarn, the language of buying and selling comes secondary to affective language: loving yarn, loving fibres, being "a lovely sponsor" and similar are all examples of Ravelry's own descriptions and justifications for promoting a particular store, designer or yarnie. This loving language is perhaps supposed to dissuade any fears that Ravelry is just like any other commercial site; commerce is only incorporated because it is a financial necessity, and Ravelry is seen as holding its sponsors, vendors and advertisers to a high, yarn-loving standard. But rather than understanding economy as a 'necessary evil' (consistent with the views of Jack Bratich discussed previously), the culture surrounding brands and yarn as commodity suggests that this facet of commodity culture is actually important to the site's users.

\section{Incorporating materiality: Ravelry's contribution to theory}

As Ravelry demonstrates, the materials purchased and the finished objects produced by Ravelers are often firmly rooted in a more recognizable consumer culture. Yarns and knitting needles in particular serve as markers of status based on the Ravelry community's perception of the value of different brands and manufacturers. Very few of the site's members appear to be driven entirely by the quest for financial gain, yet hierarchies of commodity exist, for example, in the form of 'favouriting' systems of commercial yarn and patterns. Entrepreneurship is encouraged, at times enforced, and a solid marketplace amongst Ravelers exchanging knitting goods has been visible since the site's launch in 2007. Markets (virtual or otherwise) permeate the site, yet it is not primarily a site for buying and selling. What is of interest in this apparent contradiction between commercial and non-commercial space is how commodity, affect and social capital are intertwined. These complex relationships are not adequately accounted for in existing social network literature. Similarly, interpretations of top-down capitalism influencing social interaction by creating tension between compliance and resistance fall short in an environment as multi-faceted as Ravelry's.

So where do Ravelry's social spaces and labour spaces intersect with the marketplace? And why is this intersection important? As seen in this chapter, the 'stash' and 'projects' tabs in "My Notebook" provide clear examples of where labour, affect and 
commodity overlap. This overlap is important, as it highlights the complexities of an enmeshed or entangled social reality online, where Ravelers do not need to distinguish affect from commodity exchange in order to derive meaning from the work they do both on- and offline for the benefit of the social network. Both the affective and the commoditized therefore contribute to the way labour is made meaningful on Ravelry. As a textual object, the site could easily be evaluated and explained only in terms of its social functions and its showcasing of user-generated content and labour, with the more conventional marketplace treated separately as non-essential for the site's social functionalities. Following Bratich's theory, the presence of the marketplace would be an aside or even an obstacle, unnecessary for the continuation of the "occulted labour" (313) of crafting, but present because the conventional mode of value-production happens through capitalist ideology. In other words, the marketplace is there because Ravelers have not yet broken free from it. Architecturally, commercial space is easy to single out in the site's structure, and therefore also easy to eliminate as stand-alone, not necessarily relevant to the site's social dimensions. Yet, the social spaces, labour spaces and marketplace on Ravelry all seem to act in cohesion to create a socially rewarding experience for the Ravelers themselves (Humphreys "Micro-economies" 1). Therefore, Ravelry shows no indication of wanting or needing to break away from the marketplace, as it has become absorbed and entangled within this networked community.

What Ravelry adds to the discussion of labour, affect and commerce within social networks is a very tangible materiality where "user-generated content" spills over into the offline world. It is unclear, perhaps even irrelevant, what is produced for the social network and what is produced for the offline or "IRL" (in real life; common internet slang used to describe everything which takes place in a physical, bodily reality, outside the computer). ${ }^{15}$ The notion that user-generated content blurs the lines between producer and consumer (Terranova 2000) is familiar by now, so looking only at the connection between the labour users put into the site and the site's commercial success would not necessarily contribute anything new to the on-going debates surrounding the significance of the 'prosumer' (Tapscott 1995). Similarly, looking only at the relationship between labour and social capital seems incomplete. A knitter's domestic life is undoubtedly shaped not only by the hours and hours spent on manual labour, but on acquiring, owning and storing a 'stash' of yarn and other materials. A knitter's online 
life is shaped by her commitment to documenting and displaying her manual labour the continued investment in producing "immaterial" content for the social network (requiring even more work) feeds manual production, and vice versa. This leaves a trail of finished objects, and these objects are created from commodities. It therefore makes little sense to treat affect, handicrafts and social networks as stand-alones, removed from or existing in spite of commodity and consumer culture.

\section{Conclusion}

Looking at the relationship between handicrafts and digital media is relatively new in academic circles. Existing literature on the subject varies greatly in scope, focus and conclusions. The significance of affect and care-work in capitalist society is a recurring theme within the scholarly work drawn upon throughout this project, but little consensus about the meaning and consequence of handicrafts communities can be found. The "glossing over" of capitalist ideologies and commercialism within handicrafts literature poses some challenges for evaluating the commercial space on Ravelry, as craft-work's historicity ties it to ideas of care-work, gift-giving and nurturing, nonmonetized labour. Treating these as nobler ideals underlying the revival of handicrafts ignores the entrepreneurial, profit-seeking spirits also prevalent on Ravelry. It also ignores the significance of economic transactions in shaping Ravelry's social world. Ravelers knit themselves into being not only through their offline, manual production, but through the commodity acquisition underlying this labour. The evidence does not support a "process of overcoming capitalism from the inside" (Barbrook in Terranova 36). As Sal Humphreys has already observed, Ravelry instead offers an example of hybridity "where financial and social economies co-exist" ("Economies" 234). As demonstrated in this project, Ravelry illustrates that it is possible to adopt a similar approach to understanding labour. Using this approach of co-existence has ramifications for how affect is understood and talked about in relation to new media technology. The warm, fuzzy world of crafting online may not be any more utopian, precapitalist, cyberfeminist or socially just than any other social network where the quest for social capital is perpetual. But rather than being an example of a corrosive status quo, Ravelry offers a sophisticated example of the coming together of economies in a way which forms a social whole. 
This whole is not without its problems - Tiziana Terranova, in her oft-cited "Producing culture for the digital economy" (2000), questions the punishing labour requirements of the professionals producing the digital media we all benefit from, while acknowledging that this labour holds social and personal meaning for its producers that goes beyond simple "capitalist exploitation" (33). Perhaps it is time to cast this net wider. As the boundaries between producer and consumer continue to blur and the positive relationship between social capital and social media participation has become cemented, ${ }^{16}$ so the labour investment required for successful social network participation has increased. Are we, as Ryan Bigge (2005, n. pag.) speculates, at a point where people's offline, daily lives suffer negative consequences if they do not participate in social networks, and do not invest labour in building social capital online? Ravelry demonstrates a relationship to labour similar to the tech workers discussed by Terranova, but here it is the end users of social networks who invest countless hours and precious resources into what they perceive as the bare necessities for successful relationship-building and accumulation of social capital. 


\section{Overall conclusions}

Ravelry demonstrates the need for a discussion of social media participant labour which goes beyond the immaterial and the affective. Through its reliance upon manual labour and material objects for social capital gain, Ravelry highlights the problems with understanding social networks as disembodied and immaterial. It also complicates notions of handicrafts-oriented communities as primarily affective and "pre-capitalist" (Bratich 316) by intertwining affective meanings with commodity consumption and trend. What emerges from Ravelry's use of 'finished objects' as representing a particular user is an emphasis on offline, manual labour and commodity consumption which in turn serves as entry tokens into the social network. Moreover, within the logic of Ravelry, a hand-knitted sock is not a sock until it has been photographed and catalogued in its creator's Notebook, perhaps to be viewed and commented upon by someone else. Evidence of creation and commodity acquisition must be present for successful participation in the network, and manual labour must be mirrored in a database entry in order to render the Raveler visible for validation. This is perhaps the site's core purpose - the sock isn't fully "done" until it has been shared with and confirmed by other Ravelers. This confirmation of the sock then serves as a confirmation of the Raveler and contributes to her accumulation of social capital. Posting anything at all on Ravelry is superfluous as far as creating the physical sock is concerned, but by supplying evidence of the finished objects and all its affective and commercial relations, the Raveler makes herself manifest in the eyes of the online community.

The large amount of work needed for participating on successful specialized networks like Ravelry as well as more generalized networks like Facebook, suggests that this is labour many social media participants are more than willing to carry out. Their reward is increased social capital and strengthened ties with their online community. In order to fully reap these rewards, the Raveler must adhere to a complex set of community norms in which manual work, commodity consumption and displays of affective connections all contribute to the overall textual representation of the self without becoming contradictory. Where Jenny Sundén's MUD users could represent themselves entirely through writing without needing to supply evidence of the validity of their textual performance (Digital Borderlands 2002), the Raveler's offline investment 
of time and labour underlying the performance of self online far exceeds the textual. Affect, trend and consumption all work together to support and amplify this textual performance, demonstrating that these categories are not as irreconcilable as previously thought. There is little evidence that handicrafts carried out for the purpose of participating on Ravelry is a form of subversive resistance (Minahan and Cox 11). Nor is there any evidence to suggest that Ravelers perceive commodity consumption, entrepreneurship and financial transactions as contradictory to the site's more affective spaces. This merging of social space, labour space and marketplace demonstrates that Ravelry's spaces of interaction are interdependent, neither fully affective nor fully commercial, but a synesthetic intertwining of complex categories.

Within scholarly work, different aspects of online labour are often discussed for the purpose of identifying and defining online labour types, usually with reference to the immaterial and disembodied, while less attention is paid to the sheer volume and variety of work required in order to build and maintain social capital online. As reliance upon social network technologies continues to increase, this participant labour, which far exceeds the immaterial and affective, needs to be illuminated. Tiziana Terranova (2000) and Rosalind Gill $(2002,2007)$ both observe how new media workers - the producers and maintainers of new media technologies and environments - are willing to invest more hours and more physical as well as mental resources into their work for less reward than similarly skilled workers in more traditional industries. However, similar analyses and critiques of the labour of social media participation do not appear to exist. This tendency to leave social media labour unchallenged seems to be mirrored amongst the end users of social network technologies themselves. Not only do Ravelers perform time-consuming, repetitive and at times physically straining offline tasks for the benefit of belonging within the social network, they also to an extent let community norms determine their consumption of commodities. This reveals a complex relationship between labour, commodity and social network which does not appear to have been fully addressed in existing scholarship. Ravelry offers insight into this complex enmeshing of spaces previously thought extinct, and opens up possibilities for broader analyses of social media labour. 
${ }^{1}$ As hyperlinks to forums are expressed in a manner unique to each Raveler, it is not possible to supply a
direct URL to the forums. If logged in to Ravelry, the link is expressed as
http://www.ravelry.com/discuss?set=your-boards
2 See for example Flickr, where members often record their daily lives through photographs. The act of
carrying out photography-related labour is on display as much as the photos themselves.
${ }^{3}$ It should be noted that Etsy frequently comes under fire for not adhering to its own policy of being "the
world's handmade marketplace" (etsy.com "about/team/"). The authenticity of products marketed as
handmade on Etsy is often called into question, and re-selling of wholesale mass-produced objects with or
without minor modification is common. The blog Regretsy (regretsy.com) is devoted to exposing the
perceived hypocrisy of Etsy, with frequently round-ups of Etsy listings found to be re-selling
commercially produced items. The vehemence of Regretsy followers' disdain for Etsy's reluctance to
punish those who do not adhere to the handmade-only policy demonstrates a strong attachment to the
ideological side of DIY, where "labours of love" are thought to be worth paying for but commercially
manufactured goods are not. It should be noted Regretsy pays equal attention to unappealing, confusing
or downright inadvisable examples of actual handmade goods - their slogan is "where DIY meets WTF"
(regretsy.com).
' Kate Crawford (2009) proposes to replace the pejorative term 'lurker' with the more constructive term
'listener' in the context of social media, as these listeners make up the majority of members on most social network sites. In "Following you: Disciplines of listening in social media" she argues it seems

counterproductive to dismiss silent members with dyslogistic language as their presence is arguably as important as that of content-producing members.

${ }^{5}$ MUD stands for Multi-User Dungeons or Multi-User Domains, a type of social space often used for online roleplaying games.

${ }^{6} \mathrm{~A}$ permalink is a hyperlink which remains unchanged even though the content it points to has been archived and/or moved away from its original location.

7 While futurist Alvin Toffler is typically credited with coining the term 'prosumer' in his 1980 book The

Third Wave, Canadian business author Don Tapscott re-purposed the term in a number of works

including The Digital Economy (1995) and Wikinomics (2007). It is Tapscott's more recent interpretation of the 'prosumer' that is typically encountered in modern scholarly work.

${ }^{8}$ Another distinction between Craftster and Ravelry is Craftster's reliance on irony: ironic consumption and ironic labour are visible everywhere, which contrasts with Ravelry's more earnest eagerness to provide help, support and resources for their members without much use of ironic, sarcastic or flippant language. At the time of writing, Craftster's slogan is even "No Tea Cozies without irony" (craftster.org), implying a certain distancing from the traditional, homemade object. This is not to say that Ravelry is by any means an irony-free environment, but the focus is primarily on knitting and crochet and not on being "indie".

${ }^{9}$ As the vast majority of Ravelers are women, and gender plays a prominent, if rarely commented upon, role within the site's social dynamics, Ravelers are referred to as 'she' and 'her' throughout this project. ${ }^{10}$ Hip-domestics.livejournal.com previously referred to itself as "the original" domesticity community, but as at 1 April 2012 this sub-header no longer appears. Whether the community intended to describe itself as the original domesticity community on LiveJournal, or on the internet in general, is unclear.

${ }^{11}$ As it is considered unethical to reveal usernames of people who are unknowingly being studied for academic purposes on the Internet, I am unable to provide a direct link here as this would reveal the username and personal journal of this particular LiveJournal member.

12 Somewhat ironically, Railla is often credited with coining the term "new domesticity" (Bratich 308).

${ }^{13}$ URLs to the 'stash' are of course unique to each member, but can be expressed in a template as http://www.ravelry.com/people/exampleusername/stash where "exampleusername" can be replaced with the username of any Raveler.

${ }^{14}$ Not a real yarnie

15 See, for example, the distinction between "lol" and "lol irl" where "lol" stands for "laugh out loud" and is used to acknowledge that something was humorous enough to warrant laughter, even if this laughter did not physically take place, while "lol irl" stands for "laugh out loud in real life" and means that the person who "lol'ed irl" physically laughed.

${ }^{16}$ See for example Ellison et al.'s 2009 study "The Benefits of Facebook "Friends"” where a positive correlation between Facebook use and social capital is demonstrated. 


\section{Works Cited}

Abinante, Liz. "Traveling Woman". 2009. feministy.com. n.p. Web. 7 September 2012. <http://feministy.com/traveling-woman/>.

Alison. "Happy Domesticity". 2011. n. pub. Web. 11 September 2012.

$<$ http://happydomesticity.wordpress.com/>.

Anderson, Terry, and Heather Kanuka. E-research: methods, strategies, and issues.

Ed. Burvikovs, Arnis E. Boston, MA: Allyn and Bacon, 2003. Print.

Audra Michelle (no surname given). "Redescovering Domesticity". 2010. n.

pub. Web. 19 September 2012. <rediscoveringdomesticity.com>

Banks, John, and Sal Humphreys. "The Labour of User Co-Creators." Convergence: The Journal of Research into New Media Technologies 14.4 (2008): 401-18. Print.

Bianco, Jamie Sky. "Social Networking and Cloud Computing: Precarious Affordances for the "Prosumer"." Women's Studies Quarterly 37.1/2 (2009): 303-12. Print.

Bigge, Ryan. "The cost of (anti-) social networks: Identity, agency and neo-luddites'." First Monday 11.12 (2006). N. pag. Web. <Firstmonday.org>.

Boyd, Danah M. "Friends, Friendsters, and Top 8: Writing community into being on social network sites." First Monday 11.12 (2006). Print.

Boyd, Danah M. "Why youth (heart) social network sites: The role of networked publics in teenage social life." Youth, Identity and Digital Media. Ed. Buckingham, D. Cambridge, MA: MIT Press, 2008. Print.

Boyd, Danah M., and Nicole B. Ellison. "Social Network Sites: Definition, History, and Scholarship." Journal of Computer-Mediated Communication 13.1 (2007): 210-30. Print.

Bratich, Jack. "The digital touch: Craft-work as immaterial labour and ontological accumulation." Ephemera 10.3/4 (2010). Print.

Bratich, Jack and Heidi Brush. Craftivity Narratives: Fabriculture, Affective Labor, and the New Domesticity. 2007. International Communication Association. Print.

Brocket, Jane. The Gentle Art of Domesticity: Stitching, Baking, Nature, Art \& the Comforts of Home. Berkshire: STC Craft/A Melanie Falick Book, 2008. Print.

Bury, Rhiannon. "Book review: Digital Borderlands." Canadian Journal of Communication 29.2 (2004). Print. 
Campbell, Colin. "The Craft Consumer: Culture, craft and consumption in a postmodern society " Journal of Consumer Culture 5.1 (2005): 43-64. Print.

Castells, Manuel, ed. The network society : a cross-cultural perspective. Cheltenham, UK/Northampton, MA.: Edward Elgar Pub, 2004. Print.

Chavez, Deborah J., Rich Courtright, and Ingrid Schneider. "Over the River and through the Woods." Parks \& Recreation 39.4 (2004): 68-72. Print.

Cilley, Marla. "FlyLady.net". 2012. FlyLady and Company, Inc. 18 September 2012.

"Convergence: the journal of research into new media technologies.Convergence (London, England)." (1995). Print.

Coté, Mark, and Jennifer Pybus. "Learning to Immaterial Labour 2.0: MySpace and Social Network." Ephemera 7.1 (2007): 88-106. Print.

Cowan, Ruth Schwartz. More Work For Mother: The Ironies Of Household Technology From The Open Hearth To The Microwave. New York: Basic Books, 1983. Print.

Crawford, Kate. "Following you: Disciplines of listening in social media." Continuum: Journal of Media \& Cultural Studies 23.4 (2009): 525-35. Print.

de Certeau, Michel, Luce Giard, and Pierre Mayol. The practice of everyday life volume 2: Living and cooking. Vol. 2. Minneapolis: University of Minnesota Press, 1998. Print.

Dowling, Emma, Rodrigo Nunes, and Ben Trott. "Immaterial and Affective Labour: Explore." Ephemera 7.1 (2007): 1-7. Print.

Ellison, Nicole B., Charles Steinfield, and Cliff Lampe. "The Benefits of Facebook "Friends:" Social Capital and College Students' Use of Online Social Network Sites." Journal of Computer-Mediated Communication 12.4 (2007): 1143-68. Print.

"Facebook.com". 2007. Facebook, Inc. Web. 19 September 2012.<facebook.com> Fielden, Ned L., and Maria Garrido. Internet research: theory and practice. Jefferson, N.C.: McFarland \& Co, 1998. Print.

Finin, Tim, et al. "Social networking on the semantic web." The Learning Organization 12.5 (2005): 418-35. Print.

Flew, Terry, and Stuart D Cunningham. "Creative Industries After the First Decade of Debate." The Information Society 26.2 (2010). Print.

Forbes, Jessica, and Casey Forbes. "Ravelry.com". 2007. Ravelry, LLC. 19 September 2012. 
Fornäs, J. et al, ed. Digital Borderlands: cultural studies of identity and interactivity on the Internet New York: Peter Lang, 2002. Print.

Gajjala, Radhika, and Annapurna Mamidipuni. "Gendering Processes within Technological Environments: A Cyberfeminist Issue " Rhizomes 4.Spring (2002). Print.

Garton, Laura, Caroline Haythornthwaite, and Barry Wellman. "Studying Online Social Networks." Journal of Computer-Mediated Communication 3.1 (1997): 0-0. Print.

"Geocaching.com". 2000. Groundspeak, Inc. Web. 19 September 2012. <geocaching.com>

Gill, Rosalind. "Cool, creative and egalitarian? Exploring gender in project-based, new media work in Europe." Information, Communication \& Society 5.1 (2002): 70-89. Print.

Gill, Rosalind. Technobohemians or the new Cybertariat? New media work in Amsterdam a decade after the Web. Amsterdam: Institute of Network Cultures, Hogeschool van Amsterdam, 2007. Print.

Gregg, Melissa. "The Normalisation of Flexible Female Labour in the Information Economy." Feminist Media Studies 8.3 (2008): 285-99. Print.

Hardt, Michael, and Antonio Negri. Empire. Cambridge, MA: Harvard University Press, 2000. Print.

Hardt, Michael, and Antonio Negri. Multitude: War and Democracy in the Age of Empire. New York: The Penguin Press, 2004. Print.

Hayes, Shannon. Radical Homemakers: Reclaiming Domesticity from a Consumer Culture. Left to Write Press, 2010. Print.

Haythornthwaite, Caroline. "Social networks and Internet connectivity effects." Information, Communication \& Society 8.2 (2005): 125 - 47. Print.

Hine, Christine, ed. Virtual methods: issues in social research on the Internet. Oxford/New York: Berg, 2005. Print.

"Hip Domestics". 2012. LiveJournal.com. SUP Media. Web. 28 August 2012. <hipdomestics.livejournal.com>

Humphreys, Sal. "Grassroots creativity and community in new media environments: Yarn Harlot and the 4000 knitting Olympians." Continuum: Journal of Media \& Cultural Studies 22.3 (2008): 419-33. Print.

Humphreys, Sal. "The economies within an online social network market : A case study of Ravelry." ANZCA 09 annual conference: Communication, Creativity and Global Citizenship. Ed.: Queensland University of Technology. Print. 
Humphreys, Sal. "Unravelling Intellectual Property in a Specialist Social Networking Site." Media in the Ubiquitous Era: Ambient, Social and Gaming Media. Ed. Lugmayr, Artur et al. Tampere: IGI Global, 2011. Print.

Kalin, Rob et al. "etsy.com". 2005. Etsy, Inc. 19 September 2012.

Kramer, Leah et al. "craftster.org". 2003. Internet Brands, Inc. 19 September 2012.

Lazzarato, Maurizio. "Immaterial labour." Radical Thought in Italy: A Potential Politics. Eds. Hardt, Michael and Paolo Virno. Minneapolis: University of Minneapolis press, 1996. 133-47. Print. Accessed online at <http://www.generationonline.org/c/fcimmateriallabour3.htm>.

Lee, Hyonjoo and Junghee Lee. Computer Mediated Communication Network: Exploring the Linkage Between Online Community and Social Capital. International Communication Association Annual Meeting. 2006. International Communication Association. Print.

Liu, Hugo. "Social Network Profiles as Taste Performances." Journal of ComputerMediated Communication 13.1 (2007): 252-75. Print.

Lovink, Geert. Zero comments: blogging and critical Internet culture New York: Routledge, 2008. Print.

Martens, Marianne. "Transmedia teens: Affect, immaterial labor, and user-generated content." Convergence: The Journal of Research into New Media Technologies 17.1 (2011): 49-68. Print.

Matchar, Emily. New Domesticity: Thoughts about women and homemaking in the 21st century. Ed. Matchar, Emily, 2012. Web. 12 September 2012. <newdomesticity.com> McKeon, Michael. The Secret History of Domesticity: Public, Private, and the Division of Knowledge Baltimore, 2005. Print.

Minahan, Stella, and Julie Wolfram Cox. "Stitch'nBitch: Cyberfeminism, a Third Place and the New Materiality "Journal of Material Culture 12.1 (2007): 5-21. Print.

Morini, Cristina. "The feminization of labour in cognitive capitalism." Feminist Review 87.1 (2007): 40-59. Print.

Nargi, Lela. Knitting Around the World: A Multistranded History of a Time-Honored Tradition. Minneapolis: Voyageur Press, 2011. Print.

O’Hara, Kenton. Understanding Geocaching Practices and Motivations. CHI 2008 - On The Move. April 5-10 2008. Association for Computing Machinery. Print.

Paasonen, Susanna. "Labors of love: netporn, Web 2.0 and the meanings of amateurism." New Media \& Society 12.8 (2010): 1297-312. Print. 
Poster, Mark. "Workers as cyborgs: labor and networked computers." Community in the digital age : philosophy and practice. Eds. Feenberg, Andrew and David Barney. Lanham, MD: Rowman \& Littlefield, 2004. Print.

Press, Andrea, and Sonia Livingstone. "Taking audience research into the age of new media: old problems and new challenges." Questions of Method in Cultural Studies. Eds. White, Mimi and James Schwoch. Malden, MA: Blackwell Pub., 2006. Print.

Railla, Jean. Get crafty: hip home ec. New York: Broadway Books, 2004. Print.

Rettberg, Jill Walker. "'Freshly Generated for You, and Barack Obama': How Social Media Represent Your Life." European Journal of Communication 24.4 (2009): 451-66. Print.

Ritzer, George, and Nathan Jurgenson. "Production, Consumption, Prosumption: The nature of capitalism in the age of the digital 'prosumer'." Journal of Consumer Culture 10.1 (2010): 13-36. Print.

Ross, Andrew. "The Political Economy of Amateurism." Television \& New Media 10.1 (2009): 136-37. Print.

Schlatter, Barbara Elwood, and Amy R Hurd. "GEOCACHING: 21st-Century Hide-andSeek." Journal of Physical Education, Recreation \& Dance 76.7 (2005): 28. Print.

Shacochis, Bob. Domesticity: A Gastronomic Interpretation of Love. Penguin, 1995. Print.

Shaffer, Allyson. "Online Tactics of Procrastination: Free Labor or Affective Resistance?" Conference Papers -- National Communication Association (2008): 1. Print.

Stoller, Debbie. Stitch 'n Bitch: The Knitter's Handbook. New York: Workman Publishing Company Inc, 2003. Print.

Sundén, Jenny. "Cyberbodies: Writing Gender in Digital Self-Presentations." Digital Borderlands. Ed. Fornäs, J. et al. New York: Peter Lang, 2002. Print.

Tapscott, Don. The Digital Economy: Promise and Peril In The Age of Networked Intelligence. New York: McGraw-Hill, 1996. Print.

Tapscott, Don. Wikinomics: how mass collaboration changes everything. London: Atlantic Books, 2007. Print.

Terranova, Tiziana. "Free labor: Producing culture for the digital economy." Social Text 18.2 (2000): 33-58. Print.

Wacquant, Loic J. D. "Symbolic Violence and the Making of the French Agriculturist: An Inquiry into Pierre Bourdieu's Sociology." Australia and New Zealand Journal of Sociology 23 (1987): 65-88. Print. 
Wei, Carolyn. "Formation of norms in a blog community". 2004. Into the blogosphere: Rhetoric, community and culture of Weblogs. Ed. Gurak, Laura. J. et al., 2012. Web. 19 September 2012. <http://blog.lib.umn.edu/blogosphere/formation_of_norms.html>. White, Mimi, and James Schwoch, eds. Questions of method in cultural studies. Malden, MA: Blackwell Pub., 2006. Print. 